\title{
The New Approaches Based on Ant Colony Optimization and Greedy Search Algorithm for Solving Hierarchical Chinese Postman Problem with Stochastic Travel Times
}

\section{Özlem Çomaklı Sökmen ( $\square$ ozlem.sokmen@erzurum.edu.tr)}

Erzurum Technical University: Erzurum Teknik Universitesi https://orcid.org/0000-0001-8765-0038 mustafa yılmaz

Ataturk University: Ataturk Universitesi

\section{Research Article}

Keywords: Meta-heuristic algorithm, optimization, arc routing problems, hierarchical Chinese postman problem, chance-constrained stochastic programming, new efficient algorithm

Posted Date: January 11th, 2022

DOI: https://doi.org/10.21203/rs.3.rs-1090620/v1

License: (c) (i) This work is licensed under a Creative Commons Attribution 4.0 International License.

Read Full License 


\title{
The New Approaches Based on Ant Colony Optimization and Greedy Search Algorithm for Solving Hierarchical Chinese Postman Problem with Stochastic Travel Times
}

\author{
Özlem Çomaklı Sökmen ${ }^{1}$ * and Mustafa Yılmaz ${ }^{2}$ \\ ${ }^{1}$ Erzurum Technical University, 25050, Erzurum, Turkey \\ ${ }^{2}$ Department of Industrial Engineering, Ataturk University, 25240, Erzurum, Turkey \\ *Corresponding Author: Özlem Çomaklı Sökmen. Email: ozlem.sokmen@erzurum.edu.tr
}

\begin{abstract}
Hierarchical Chinese postman problem (HCPP), a variant of the Chinese postman problem, aims to find the shortest tour or tours by passing through the arcs classified according to precedence relationship. HCPP, which has a wide application area in real-life problems such as shovel snow and routing patrol vehicles where precedence relations are important, belongs to the NP-hard problem class. In real-life problems, travel time between the two locations in city traffic varies due to reasons such as traffic jam, weather conditions, etc. Therefore travel times are uncertain. In this study, HCPP is handled with the chanceconstrained stochastic programming approach, and a new type of problem, hierarchical Chinese postman problem with stochastic travel times, is introduced. Due to the NP-hard nature of the problem, the developed mathematical model with stochastic parameter values cannot find proper solutions in large size problems within the appropriate time interval. Therefore, two new solution approaches, a heuristic method based on the Greedy Search (GSA) algorithm and a meta-heuristic method based on ant colony optimization (ACO) are proposed in this study. These new algorithms were tested on modified benchmark instances and randomly generated problem instances with as many as 817 edges. The performance of algorithms was compared in terms of solution quality and computational time.
\end{abstract}

\section{KEYWORDS}

Meta-heuristic algorithm; optimization; arc routing problems; hierarchical Chinese postman problem; chance-constrained stochastic programming; new efficient algorithm.

\section{Introduction}

The goal of the arc routing problems is to establish the shortest tour or tours that return to the starting point by stopping at least once at all arcs on a network. If all arcs/edges ought to be visited in this network and the shortest route or routes are to be identified, the problem called the Chinese Postman Problem (CPP) while the shortest tour or tours are should be introduced by visiting only certain arcs/edges, the problem is called the Rural Postman Problem (RPP) (Corberan et al. 2002).

CPP and RPP each have variations for different uses and assumptions. In this study, Hierarchical CPP (HCPP), which takes into account the precedence relationship between the arcs is discussed. The most typical real-life examples of HCPP are activities such as garbage collection, street cleaning, snow shoveling, road maintenance, and repair which take into account the precedence relationships between roads. HCPP attempts to find the optimal tours that traverse each arc in the offered network at least once, taking into account precedence relations of the paths roads $(\operatorname{arc})$ in a given network. 
Stochastic Vehicle Routing Problem (SVRP) occurs when one or more deterministic Vehicle Routing Problem (VRP) parameters are random. Because of the uncertainty, it includes SVRP is more similar to real-life problems. Customers, inquiries, travel times, or service times may be random elements of this sort of issue. In the present era where the competitive environment is at its peak, it is necessary to know the travel times between nodes in order not to lose time for corporations in the public and private sectors and to avoid the reduction in the quality of the services and enable accurate planning. It is an undeniable issue that optimal route planning needs to be generated by taking factors such as distance and time into consideration which are deemed essential for companies and customer satisfaction procedure. Yet, owing to unpredictable weather conditions, environmental circumstances, or traffic, it is not often feasible to articulate travel times and speeds with exact values. On the very same day, although the travel times rely on the distance, it may be stochastic due to accidents or multiple factors that hinder transport. In the literature, this form of problem, which was first discussed by Kao (1978) expresses the stochastic state of traffic, is referred to as vehicle routing problem with stochastic Travel Times (STT-VRP) or vehicle routing problem with Stochastic Service Times (SST-VRP).

Concerning the literature research, most HCPP-related studies have used the distances while the arrival times, which differ due to uncertainties have not taken into consideration. However, in actual life in urban traffic, the travel time between nodes is not constant owing to changing traffic intensity and weather conditions. Because of this uncertainty, HCPP has been solved within the scope of this study by using the chance-constrained stochastic programming method. Thus, it is shown that the STT-HCPP which is addressed within the scope of this paper, is a different form of problem that has never before been observed. In particular, a mathematical model for HCPP has been established in prior studies, and it can be stated that the mathematical model formed due to the problem's NP-hard nature offers suitable solutions only for smallsized problems. Two new algorithms, one heuristic, and the other meta-heuristic were suggested within the framework of this study, to determine the best answer to the problem in a reasonable time. It is also critical that the proposed new methods are focused on the chance-constrained stochastic approach. It is aimed at the problem type and suggested solution approaches to adapt HCPP to real life and to be the first study in the literature.

Briefly, the two main scientific contributions of this paper are described below.

1. This research is the first study to solve HCPP using a chance-constrained stochastic programming approach while taking into account the stochasticity of travel times in HCPP and as well as assuming that these parameters are appropriate for a certain distribution. In several of the VRP and Traveling Salesman Problem (TSP) studies (Taş et al. 2013; Tadei et al. 2017), which are the most general implementation areas of node routing problems before (Taş et al. 2013; Tadei et al. 2017), travel times and/or costs are regarded as stochastic. However, there is no similar study for HCPP which is an edge routing problem. A new STTHCPP problem form was therefore introduced for the first time in this study.

2. In this study, two new algorithms are proposed for the solution of large-size STT-HCPP problems, which are easier to apply to real-life problems owing to uncertainty it includes. The first is the heuristic method based on the Greedy Search (GS) algorithm, while the second is a meta-heuristic method based on ant colony optimization (ACO). The purpose of these methods is to find solutions to large-size problems in a brief time. Algorithms are tested and compared for their effectiveness by using these methods for test problems.

\subsection{Literature Survey}

In this subheading, firstly, studies related to HCPP in the literature are discussed, and then studies related to stochastic parameters of CPP, STT-SVRP, and STT-TSP are examined, respectively.

HCPP was first proposed by Dror et al. (1987) by addressing a network of arcs divided into classes in which precedence relationships are described. They demonstrated that the issue can be resolved in polynomial time if the precedence relationships are linear and all sub-graphs are entangled in the hierarchy classes. In general, they proved that the problem is in the NP-hard class. Alfa and Liu (1988) addressed a 
more rational scenario in which subgraphs are not entangled in hierarchical classes. In their study, they proposed an approach to the heuristic solution for HCPP that can be especially used in snow shoveling. Damodaran (2008) developed a new heuristic method for the combination of disconnected hierarchical classes. It was claimed that this method established in the study accomplished better results than the heuristic model of Alfa and Liu (1988). Ghiani and Improta (2000) have suggested an algorithm that needs less computational work and that offers a clear solution in a shorter period than earlier studies. In this study, the first algorithm which provides a precise solution for HCPP is discussed. In their research, Cabral et al. (2004) had established a method for converting the HCPP into an equivalent Rural Postman Problem. They had suggested a splitting heuristic for unidirectional HCPP with linear precedence relations. The converted problem was subjected to a branch-and-cut algorithm to overcome HCPP which has two objectives which are the hierarchical purpose and production time. In the study, Korteweg and Volgenant (2006) developed an existing algorithm that solves HCPP in their work and they reduced the time complexity of the original algorithm with their optimistic technique to the $\mathrm{O}\left(\mathrm{kn}^{4}\right)$ (where $\mathrm{k}$ is the number of layers in the graph and $\mathrm{n}$ is the number of nodes). Under such an algorithm, each sub-network 's distinct minimum cost routes are determined according to precedence levels. Damodaran et al. (2008) have established a heuristic approach that offers improved lower HCPP bound values compared to CPP. With this lower bound value, it was decided that in algorithms that provide exact solutions such as branch and bound algorithms, the optimum solution is achieved in a shorter period. Xu et al. (2014) suggested a recent algorithm focused on the methodology of cell separation for efficient coverage of a defined location packed with random obstacles. First, the cells are separated into pieces in this hierarchical method, then, the CPP is resolved to determine an Euler tour that travels through these cells. In their studies Sayata and Desai (2015) proposed a new method to eliminate the time complexity of algorithm O $\left(k n^{5}\right)$ (by Dror et al. 1987) and used the Kruskal method to reduce the number of edges in a network that has a time complexity of $\mathrm{O}\left(k^{2} n^{2}\right)$. Colombi et.al. (2017) discussed the hierarchical mixed RPP in their study. A branch and cut algorithm are presented in the study which offers a certain solution to the problem, and it has been shown that the results achieved with the algorithm are more effective and efficient than other existing methods. The last study on HCPP in the literature belongs to the Çodur and Y1lmaz (2020). Authors discussed time-dependent HCPP when travel time changes according to the time of the day. A mixed-integer mathematical model for problem-solving was established in the research, and the genetic algorithm (GA) and hybrid simulated annealing were suggested as a solution approach to large-scale problems.

To the best of our knowledge, there are no studies in the literature concerned with stochastic travel times concerning HCPP. On the other hand, there is a very limited amount of studies on the stochastic parameters of CPP. Tan et.al. (2005), addressed the CPP in the studies of networks with stochastic travel times. They proposed an algorithm as a solution. Peng and Zhang (2012) have studied on three uncertain programming models for CPP with uncertain weights. They claimed that these models could be transformed into deterministic equivalent models. They used Dijkstra's algorithm and Fleury's algorithm to solve the problem. Majumder et al. (2019), have proposed multi-objective CPP under the framework of uncertainty theory for an undirected connected network. They presented an expected value model as a solution approach.

There has been no prior research on STT-HCPP, as far as we know. However, a review of the related literature indicates that research on STT-SVRP and STT-TSP are present. Kao (1978) discussed the TSP with stochastic travel time (STT-TSP) for the first time. In this research, a dynamic programming dependent heuristic algorithm is suggested for problem-solving. Carraway et al. (1989) established generalized dynamic programming for STT-TSP which even in the absence of uniformity guarantees optimum level. Laporte et al. (1992) conducted a study on stochastic travel and service time-based VRP. A banking example is examined in this study. For the issue under discussion, they proposed three separate models including the chance-constrained model. For the solution of these models, they proposed the branch and cut algorithm. Kenyon and Morton (2003) focused on SVRP on a network with random travel and service times in their study. Two stochastic programming models have been developed in the study. The first model minimizes the estimated delivery period, while the second model maximizes the likelihood of project completion at a specified period. Kim et al. (2005) addressed the topic of vehicle routing in a stochastic 
network, utilizing real-time traffic information. A decision-making process was built in this research, based on the Markov decision procedure. Jula et al. (2006) worked with the planning of truck routes within stochastic networks. A methodology for forecasting the arrival times to the customers is suggested in the process described in this study since travel and service times are uncertain. An approximate solution based on dynamic programming is provided using the estimated time of arrival. Russell et al. (2008) have studied vehicle routing with soft time windows and erlang travel times. In this research, travel times were acknowledged as suit the gamma distribution. A tabu search algorithm has been developed to solve the problem. Chang et al. (2009) studied a TSP traveling with time windows and stochastic and dynamic travel time. In the research, it is presumed that arrival times in nodes are normally distributed, and a heuristic method for solving the problem has been established. Shen et al. (2009), addressed VRP with uncertain demand and travel time parameters to mitigate unmet demand. In the study, a chance-constrained programming technique was used and a tabu search heuristic method was proposed to solve the model. Hou and Zhou (2010), suggested a stochastic programming model that would take stochastic demand and travel time into account and introduced a genetic algorithm created to solve this problem. Li et al. (2010) examined a VRP with time windows and stochastic travel and service times in their study. The problem has been modeled according to two different approaches namely the chance-constrained model and two-stage stochastic programming with recourse model. A heuristic method based on tabu search which takes into account the stochastic nature of the problem has been presented to overcome both models effectively. Errico et al. (2016), have studied VRP with hard time windows and stochastic service times. In the study, it was accepted that the service times fit symmetrical triangular distribution and the chance-constrained stochastic programming model was used. Taş et al. (2013) analyzed the VRP with stochastic travel times including soft time windows and service costs. The gamma distribution was used for travel time in the study. For the model's solution, a tabu search method was suggested. Zhang et al. (2013) addressed the issue of stochastic vehicle orientation with soft time windows under uncertain travel and service time. They introduced a new stochastic programming model to reduce the carrier's total expense, thus guaranteeing the likelihood of arrival to every customer in the minimum time. A recursive tabu search heuristic has been developed to solve the problem. Errico et al. (2016) examined VRP with hard time windows and stochastic service times. They used chance-constrained programming to model the problem. The study results indicate that the approach proposed is effective and that a stochastic chance-constrained model has benefits over deterministic models. Gutierrez et al. (2016), suggested a memetic algorithm for solving a VRP with time windows and stochastic travel and service times. The effects of the proposed Multipurpose Memetic Algorithm and the Multi-objective Recursive Local Search algorithms were contrasted and their efficacy was demonstrated. Ehmke et al. (2015), studied the problem of vehicle routing with a time window and stochastic travel times. A method for estimating the distribution of the initial service times and arrival times has been suggested in the research. The results of the research demonstrate how solutions are influenced by adjusting customer care standards, distribution of travel time, and different parameter settinGSA. Binart et al. (2016), discussed VRPs with stochastic service and travel times. In the study, the mentioned time are examined concerning the triangular distribution. The model was first approached using a branch and cut algorithm or a Lagrange parsing method, and then, a heuristic method was used to create the solution. Miranda and Conceição (2016) studied the problem of VRP with hard time windows and stochastic travel and service time in their study. Travel and service times were regarded as parameters with normal distribution in this research but it was noted that arrival times with normal distribution were not well modeled. A meta-heuristic based on Recursive Local Search is proposed for the solution of the problem. Nguyen et al. (2016) focused on solving the stochastic VRP with time windows under uncertainty. In the research, given the complicated nature of chance-constrained stochastic programming, some measures are recommended for the uncertainties in the specific problem. Suggested suitable steps to solve the problem are introduced into an existing heuristic method of tabu-search. Miranda et al. (2018), studied the problem of multi-objective VRP with hard time windows and stochastic travel time and service time.Travel and service times are modeled as normal distribution in the study. Two separate algorithms are suggested for the solution of the problem, namely the Memetic Algorithm and Recursive Local Search, and the results have been compared. Mukherjee et al. (2019), introduced some constrained covering solid TSPs in their 
study. In real-life situations, since some parameters cannot be expressed with crisp values, traveling costs are regarded as rough variables in their study. Covering distance is considered both as a fuzzy and a rough variable. A new heuristic algorithm is suggested as a solution approach. Majumder et al. (2019) discussed the uncertain multi-objective multi-item fixed charge solid transportation problem with budget constraint. In the model suggested, many variables such as transportation costs, transportation times were assumed as uncertain variables. In the uncertain model, the parameters are treated as zigzag and normal uncertain variables. Under the uncertainty theory, three different models, namely the expected value model, the chance-constrained model, and the dependent chance-constrained model have been developed. Equivalent deterministic transformations of these models have been generated and they are solved through 3 different methods, which are the fuzzy programming method, linear weighted method, and global criterion method. In their study, Dutta et al. (2019) presented a new approach to the solution of open VRP. In this new method, the Genetic Algorithm and modified Kruskal's method is hybridized. This suggested approach is adaptable to real-life cases, and the obtained results of the suggested method have been compared with evolutionary methods.

When reviewing the HCPP studies in the literature, it is found that several of these studies do not take into consideration the travel times between nodes but acknowledge the distances between nodes. In addition, there is no research on this topic that deals stochastically with travel times. In order to fill this gap in the literature in this study, HCPP with Stochastic Travel Times (STT-HCPP), which is a new problem form, is taken into account. It is agreed in the analysis that travel times, which represent randomness, are normally distributed.

\section{Preliminaries}

\subsection{Stochastic Programming}

Difficulties generally experienced while trying to determine the values of model parameters $\left(\mathrm{c}_{j}, a_{i j}, b_{i}\right)$ used in modeling real-life problems with linear programming. In this context, taking the parameter values as exact values by utilizing existing evidence or past studies is not a correct approach since it may impact the study's impartiality. Moreover, model parameters do not necessarily have exact values but are represented with random variables. Problems that contain some or all of the parameters of their model as random variables are named stochastic programming problems (SPP) (Atalay and Apaydin 2011). Concerning the Stochastic programming that George Dantzig first proposed in the 1950s, substantial improvement has since been achieved in understanding model properties and developing algorithmic methods to solve them (Higle 2005).

Stochastic programming is one of the most commonly employed techniques of routing problems, where parameter values are hard to express as exact values. And also, the amount of garbage to be collected before the vehicle reaches the specific places cannot be identified precisely in real-life issues, in a garbage collection process. Likewise, even though the vehicle is always traveling on the same road path, the duration of the travel cannot be described with exact values, since the arrival times can vary due to weather and environment. Again, the punctuality of a flight can vary depending on weather conditions.

Classic VRP is the problem of determining the optimum route/routes to serve customers in a predetermined network with a fleet of vehicles, to reduce the overall cost of traveling in comparison to travel periods or distance and operating costs based on the number of vehicles used. Consequently, it is intended to decide the lowest cost route by following the constraints defined in the VRP. SVRP occurs when some parameters of Classic VRP are random. The most common examples of this are the stochastic travel time and stochastic demands (Gendreau et al. 1996).

In which the SVRP studies are analyzed, some of the research in this area has explored creating a suitable mathematical model and approaches to solutions. In some studies, it was aimed to suggest algorithms for the solution of SVRP. Looking once again at the literature studies when building the mathematical model, it is shown that SVRP's chance-constrained stochastic programming and stochastic programming with recourse approaches are used (Oyola 2018) 
In problems that inherit random variables, the chance-constrained approach is used to determine the decision variables in a single step. Chance-constrained stochastic programming is the transformation of variables from a certain distribution of probability into a deterministic state, to the degree that the probability levels enable it, concerning the problems with random parameter values (K1zıloğlu 2017).

It is aimed at finding a first-stage solution in stochastic programming with recourse that minimizes the estimated cost of the second-stage solution. The cost here consists of the costs of the recourse along with the first stage solution's cost (Gendreau et al. 1996). In this study, chance-constrained Stochastic programming is discussed.

\subsubsection{Chance-Constrained Stochastic Programming}

First introduced by Charnes and Cooper (1959) chance-constrained stochastic programming is about revealing the parameters that are random variables of a certain distribution of probability and expressing this probabilistic framework with the function of probability in the objective function and the constraints of the problem. In this technique, the nonlinear deterministic equivalents of these chance constraints are used instead of the chance constraints of the problem and the problem is solved using recognized nonlinear programming techniques. Chance-constrained linear programming model defined as follows;

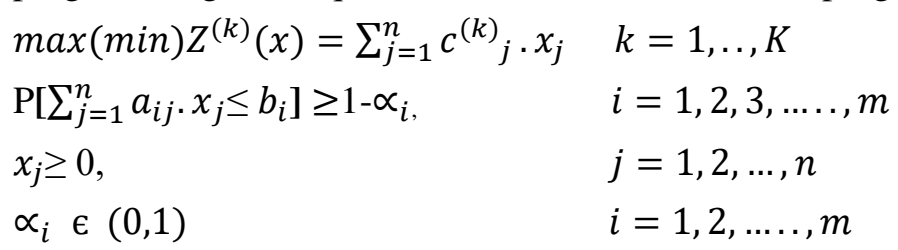

In the model, the $c^{(k)}, a_{i j}$ and $b_{i}$ are model parameters, and some or all of them are random variables. $\propto_{i}$ are the predetermined possibilities. Here decision variables of $x_{j}$ have a deterministic structure (Taha 2007). The stochastic parameters in the model must have certain distribution and probability levels of $\propto_{i}$ should be determined. The model is solved using this information by rendering it a deterministic form (Aksarayli and Pala 2015).

In the specified model provided by Eq. (1), Eq. (2), Eq. (3) and Eq. (4) each of the coefficients that are random variables should have a certain distribution or their mutual distribution should be known (Atalay and Apaydın 2011). The aim here is to solve the problem by obtaining the deterministic equivalent form of the model that have coefficients as random variables.

\subsubsection{Chance-Constrained Models whose Coefficients are Random Variables with Normal Distribution}

In linear programming problems where at least one of the coefficients of $c_{j}^{(k)}, a_{i j}$ and $b_{i}$ is a random variable, it is assumed that these coefficients generally show normal distribution characteristics. Under this assumption, in the chance-constrained stochastic linear programming problem modeled in Section 2.1 $c_{j}^{(k)}, a_{i j}$ and $b_{i}$ are random variables with a normal distribution. Determination of chance-constraints with normally distributed coefficients are shown with 7 cases defined as follows (Hulsurkar et al. 1997).

1. Only $a_{i j}$ coefficients are random variable,

2. Only $b_{i}$ coefficients are random variable,

3. Only $c_{j}^{(k)}$ coefficients are random variable,

4. Both $a_{i j}$ and $b_{i}$ coefficients are random variables,

5. Both $a_{i j}$ and $c_{j}^{(k)}$ coefficients are random variables,

6. Both $c_{j}^{(k)}$ and $b_{i}$ coefficients are random variables,

7. Both $a_{i j}, b_{i}$ and $c_{\mathrm{j}}^{(k)}$ coefficients are all random variables, 
The only parameter that can be considered stochastic in HCPP is the objective function coefficients, $c_{j}^{(k)}$, within the scope of this study the case in which "only $c_{j}^{(k)}$ coefficients are random variables" will be discussed.

Let's assume $c_{j}^{(k)}$ are random variables with a normal distribution and the $z^{(k)}$ is the kth objective function. The mean of $z^{(k)}$ is given in the form of $E\left(z^{(k)}\right)$. Here the deterministic linear programming equation referring to the problem of probabilistic linear programming is as follows:

$E\left(z^{(k)}\right)=\sum_{j=1}^{n} E\left(c_{j}^{(k)}\right) x_{j}, \quad k=1, \ldots, K$

Here, $E\left(c_{j}^{(k)}\right)$ is the mean of $c_{j}^{(k)}$. Thus, the deterministic objective function that has the expected value model (E-model) will be as follows (Hulsurkar et al. 1997).

$\max (\min )=E\left(z^{(k)}\right)=\sum_{j=1}^{n} E\left(c_{j}^{(k)}\right) x_{j}, \quad k=1, \ldots, K$

\section{Mathematical Formulation}

For the HCPP belonging to the NP-hard problem class, paths /arcs are divided into classes and a precedence relationship is defined in these classes (Dror et al. 1987). Therefore, in HCPP the order in which the paths/arcs are to be followed is set beforehand. The object of this type of problem is to establish the shortest tour/tours considering the precedence relationships and by traveling through each arc/path at least one time in a hierarchical network. Snow removal and salt dumping activities, where roads are categorized according to their importance (urban or rural, main road, secondary road, etc.) are one of the best examples that can be offered for HCPP in real life. Concerning these procedures, when the aforementioned procedures are made on the main roads of the first precedence class firstly and all the main roads are completed, we may pass to other roads.

One of the most important assumptions belonging to linear programming is the fact that model parameters have a deterministic structure. The classical mathematical model of HCPP is very useful in this context. However, these parameters take place as uncertain in most of the real-life problems. This uncertainty resulting from different factors can't be represented with mathematical models. Therefore, chance-constrained stochastic programming has been used in this study to include this uncertainty in mathematical models. Most of the data excepted as exhibiting normal distribution since it would be more suitable for real life in terms of statistics. Therefore, within the scope of this study, cij objective function coefficients that show the travel time between two nodes in HCPP are considered as random variables with normal distribution characteristics, and the model was transformed into STT-HCPP by making use of "the case where the only coefficients of cj are random variables which are explained in Section 2.1.1.1.

Let's consider a $\mathrm{G}(\mathrm{V}, \mathrm{E})$ network where $\mathrm{V}$ represents the set of nodes and $\mathrm{E}$ is a non-directional set of arcs (paths). This network is divided into $m$ different sub-networks, each of these represents a hierarchical level. In this case, a hierarchical network is shown as; $G(V, E)=G_{1}\left(V_{1}, E_{1}\right) \cup G_{2}\left(V_{2}, E_{2}\right) \cup \ldots \cup G_{m}\left(V_{m}, E_{m}\right)$ (Y1lmaz 2018). Here, $\mathrm{G}_{\mathrm{m}}$ is the mth sub-network showing the arcs/ roads in the that will be traveled on the mth order.

The indexes, sets, parameters, and decision variables used in the HCPP integer linear programming model are shown below under respective titles (Çodur and Yilmaz 2020)

Indexes;

$i, j \quad$ : nodes on the network

$t \quad$ :number of the edge traversal in the route (steps)

$h \quad$ : hierarchical levels.

$\underline{\text { Sets; }}$

$i, j / \mathrm{V}:$ Set of vertices $i, j=\{1,2, \ldots, n\}$,

$h / \mathrm{H}:$ Set of hierarchies $\{1,2, \ldots, h\}$, 
$t \leq L \quad$ : number of steps $L=2\|E\|$

$\mathrm{E}_{h} \quad:$ Set of edges of $h$ th hierarchy class

E : Set of all edges in the network, $E=\left\{\mathrm{E}_{l} \cup \mathrm{E}_{2} \cup \ldots \cup \mathrm{E}_{h}\right\}$

$\left\|E_{h}\right\| \quad:$ The total number of edges of $h$ th hierarchy class

$\delta_{\mathrm{h}}(\mathrm{i}) \quad$ : The edges of $E_{h}$ set that exist from node $i, \delta_{\mathrm{h}}(\mathrm{i})=\left\{j \mid(i, j) \in \mathrm{E}_{h}\right\}$.

$\delta(i) \quad$ : Set of all edges that exist from node $i, \delta(i)=\mathrm{U}_{h \in H} \delta_{h}(i)=\left\{\delta_{1}(i) \cup \delta_{2}(i) \cup \ldots \cup \delta_{h}(i)\right\}$

$\underline{\text { Parameters; }}$

$\mathrm{C}_{i j} \quad$ : Distance matrix of the edge $(i, j)$

$\mathrm{B}_{i j} \quad$ : Connection matrix of the edge $(i, j)$

$\mathrm{O}_{i j} \quad$ : Precedence matrix of the edge $(i, j)$

M : Is a sufficiently large number

n : The total number of nodes in the graph.

Variables;

$x_{i j}^{t}=\left\{\begin{array}{l}1 \\ 0\end{array}\right.$ if traversed from node $i$ to node $j$ at $t$ th step 1 , otherwise 0.

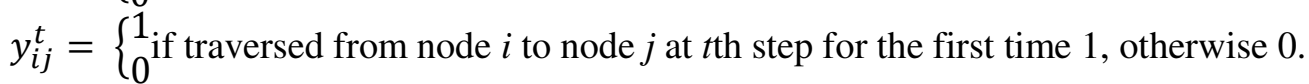

$\phi_{h t}=\left\{\begin{array}{l}1 \\ 0\end{array}\right.$ if traversed from all edges of $h$ th hierarchy in $t$ th step completed 1 , otherwise 0.

Using this information, HCPP's mixed-integer linear programming model can be written as follows (Çodur and Y1lmaz 2020). In this model; when the objective function coefficients of $\mathrm{c}_{i j}$ are considered as random variables with normal distribution characteristics, the problem transforms into STT-HCPP.

$\operatorname{Min} E(z) \sum_{i=1}^{n} \sum_{j=1}^{n} \sum_{t=1}^{T} c_{i j} x_{i j}^{t}$

Subject to;

$\sum_{t=1}^{L}\left(x_{i j}^{t}+x_{j i}^{t}\right) \geq 1 \quad \forall(i, j) \in E$

$\sum_{i} x_{i j}^{t-1}=\sum_{s} x_{j s}^{t} \quad \forall j, t \quad t>1 \quad i, j, s \in V$

$\sum_{(i, j)} x_{i j}^{1} \geq 1 \quad(i, j) \in \delta_{1}\left(i_{0}\right)$

$\sum_{\mathrm{j}} x_{j i}^{t} \geq 1 \quad t=t_{\text {end }}, i=i_{0}$

$\sum_{(i, j) \in E} x_{i j}^{t}=1 \quad \forall t$

$x_{i j}^{t}+x_{j i}^{t} \geq y_{i j}^{t} \quad \forall i, j, t \quad i<j$

$\sum_{t^{\prime}<t}\left(x_{i j}^{t \prime}+x_{j i}^{t \prime}\right) \leq M^{*}\left(1-y_{i j}^{t}\right) \quad \forall i, j, \mathrm{t} \quad i<j$

$\sum_{t=1}^{L} y_{i j}^{t}=1 \quad \forall(i, j) \in E \quad i<j$

$\sum_{t^{\prime} \leq t} \sum_{(i, j) \in E_{h}} y_{i j}^{t^{\prime}} \geq\left\|E_{h}\right\| * \phi_{h t} \quad \forall h, t$

$\sum_{t^{1} \leq t} \sum_{(i, j) \in E_{h+1}}\left(x_{i j}^{t \prime}+x_{j i}^{t \prime}\right) \leq M * \phi_{h t} \quad \forall t, h=1, \ldots, H-1$

$x_{i j}^{t}, y_{i j}^{t}, \phi_{h t} \in\{0,1\}$

$\forall i, j, t, h$

Eq. (7) expresses the deterministic objective function with the expected value model that aims to minimize the total tour time. Eq. (8) is restriction representing the traveling from all road on the graph at least once, Eq. (9) is the provision of continuity among the nodes, Eq. (10) is traveling from any one of the 
roads belonging to the $1^{\text {st }}$ hierarchy that can be traveled from the $i_{0}$ start node in the first step $(t=1)$, Eq. (11) is the return to the start node $\left(I_{0}=1\right)$ at the last step, Eq. (12) the travel from only one road in each step, Eq. (13-15) is the assignment of value to the $y_{i j}^{t}$ variable defining the step on which the $(i, j)$ arc is traveled firstly. Eq. (16) checks whether all the roads belonging to the $h$ hierarchy has been traveled from in the $t^{\text {th }}$ step. Eq. (17) shows that without completing the roads in the $h^{\text {th }}$ hierarchy, the roads of the $(h+$ $1)^{\text {th }}$ hierarchy are not passed from, and Eq. (18) presents that the road usage variables must have integer values $\{0,1\}$ (Çodur and Yilmaz 2020).

\section{Numerical Illustration}

When reviewing the literature on HCPP, two small-sized network examples belong to this type of problem. These are the networks that authors used in their studies (Dror et al. 1987; Korteweg and Volgenant 2006).

The small-sized hierarchical network developed by Dror et al. (1987) and the cluster of roads belonging to the hierarchical classes in this network which are discussed under this subheading are presented in Fig. 1 below.

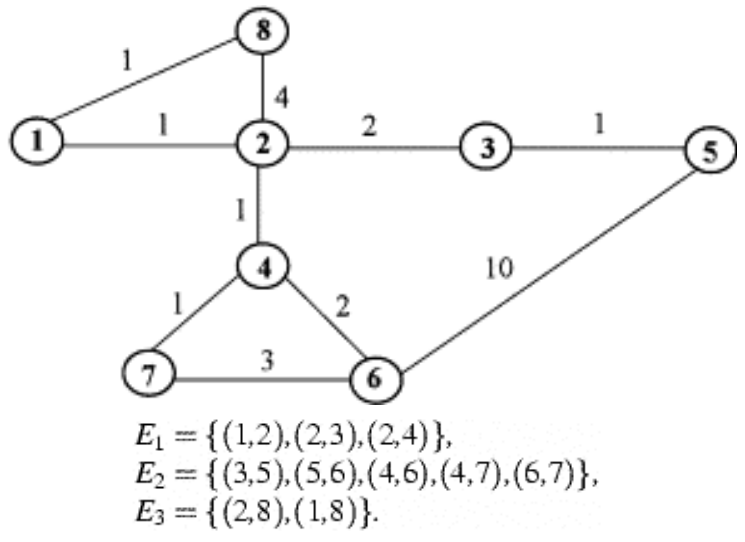

Fig. 1: Hierarchical network example

This hierarchical network contains 8 nodes as following $V=\{1,2,3,4,5,6,7,8\} . E=\left\{E_{1} \cup E_{2} U\right.$ $\left.\mathrm{E}_{3}\right\}$ represents the arc cluster belonging to each hierarchy class. Dror et al. 1987 by using this network, have determined the optimum tour distance as 30 and the optimum tour route as 1-2-4-2-3-5-6-7-4-6-4-2-8-1.

While in this study, since the arrival times are uncertain due to traffic density, weather conditions, etc., the network shown in Fig. 1 is taken into consideration as STT-HCPP. The only parameter that can be considered stochastic due to the nature of HCPP is the $c_{i j}$ coefficients. Hence, instead of distances between nodes within the network, the arrival times are used. Distance values on the network have been transformed into the arrival times based on the legal speed limit within the city. The arrival times for each road are assumed as stochastic as well as considering the traffic density (low density, normal, high density). Since it would be the most appropriate distribution for real life, arrival times within the framework of the study are assumed to meet the normal distribution characteristics. In the mathematical model of HCPP, expected values, and variances for random variables of $c_{i j}$ which are the objective function coefficients are presented in Table 1. Since the $c_{i j}$ are symmetrical $\left(c_{i j}=c_{j i}\right)$ related expected values and variances are provided in Tab. 1 only for once. For example, $c_{12}=c_{21}=1,021$.

Table 1 Expected values and variances of the coefficients of the hierarchical network sample 


\begin{tabular}{|l|l|l|l|l|l|l|l|l|l|l|}
\hline $\begin{array}{l}\text { Coefficients } \\
\left(\boldsymbol{c}_{\boldsymbol{i j}}\right)\end{array}$ & $\boldsymbol{C}_{\boldsymbol{1 2}}$ & $\boldsymbol{C}_{\boldsymbol{1 8}}$ & $\boldsymbol{C}_{\mathbf{2 3}}$ & $\boldsymbol{C}_{\mathbf{2 4}}$ & $\boldsymbol{C}_{\mathbf{2 8}}$ & $\boldsymbol{C}_{\mathbf{3 5}}$ & $\boldsymbol{C}_{\mathbf{4 6}}$ & $\boldsymbol{C}_{\mathbf{4 7}}$ & $\boldsymbol{C}_{\boldsymbol{5 6}}$ & $\boldsymbol{C}_{\boldsymbol{6 7}}$ \\
\hline $\begin{array}{l}\text { Expected } \\
\text { Value }\end{array}$ & 1,021 & 1,063 & 1,039 & 1,103 & 0,854 & 0,782 & 0,804 & 0,799 & 1,471 & 1,387 \\
\hline Variance & 0,028 & 0,028 & 0,056 & 0,028 & 0,111 & 0,028 & 0,056 & 0,028 & 0,278 & 0,083 \\
\hline
\end{tabular}

The cost matrix of the deterministic objective function that has the new expected value model developed by using Table 1 and the objective function of the STT-HCPP mathematical model are stated below. In this model, the Eq. (8-18) constraints provided in Section 3 are applied.

$$
\left[c_{i j}\right]_{8 \times 8}=\left(\begin{array}{cccccccc}
- & 1,021 & - & - & - & - & - & 1,063 \\
1,021 & - & 1,039 & 1,103 & - & - & - & 0,854 \\
- & 1,039 & - & - & 0,782 & - & - & - \\
- & 1,103 & - & - & - & 0,804 & 0.799 & - \\
- & - & 0,782 & - & - & 1,471 & - & - \\
- & - & - & 0,804 & 1,471 & - & 1,387 & - \\
- & - & - & 0.799 & - & 1,387 & - & - \\
1,063 & 0,854 & - & - & - & - & - & -
\end{array}\right)
$$

$\operatorname{Min} E(z): \sum_{i=1}^{n} \sum_{j=1}^{n} \sum_{t=1}^{T} c_{i j} x_{i j t}=(1,021) x_{121}+(1,063) x_{181}+\cdots+(0,854) x_{82 \mathrm{~T}}$

The model was solved by using CPLEX solver in GAMS 24.2.3 package program. Optimum route found as 1-2-3-2-4-6-4-7-6-5-3-2-8-1 and objective function value as $\operatorname{Min} E(z)=13.205$. As shown in this example of a small network, the route and cost value can differ significantly by utilizing stochastic travel times, rather than distance, in the objective function.

\section{Methodology}

If the size of the problem rises in routing problems, it becomes difficult to implement exact methods of solution which are difficult to calculate but which provide reasonable results. For this purpose, combinatorial optimization problems like TSP, VRP, HCPP can usually not be solved with exact methods in polynomial time, and heuristic and/or meta-heuristic methods are being applied to solve NP-hard problems.

Based on the nature of the problem being studied, heuristic algorithms are prepared, however heuristics do not always promise the optimal outcome and do not always produce the same results. In the worst-case scenario, heuristics can produce outcomes far from the optimum solution, however, welldesigned heuristics will outperform the best approximation algorithms (Uğurlu 2018). The nearest neighbor algorithm, greedy algorithms, saving algorithm are among the most commonly used heuristic algorithms. This group also is known as classical heuristic methods.

Meta-heuristic approaches are techniques developed to solve complex optimization problems that cannot be solved with exact methods, do not promise the exact solution but generate nearly optimal solutions in a short period. The key difference between meta-heuristics and classical heuristics is that metaheuristics in the solution space make an extensive and deep search and consider inadequate solutions when needed. Therefore, meta-heuristic methods can provide better quality solutions. However, to execute metaheuristics in the right way, the parameters need to be changed to fit the situation (Laporte et al. 2000). The usage of meta-heuristic approaches including genetic algorithm, tabu search algorithm, simulated annealing, ACO algorithm, etc. are frequently noted in the literature.

When the literature is reviewed, it is found that exact algorithms and heuristic methods are commonly used in the HCPP research, which is one of the combinatorial optimization problems, and meta-heuristic methods are used to solve problems in just one sample. In this paper, two algorithms, one of them based on 
the GSA Algorithm, and the other based on the ACO among heuristic methods are proposed for the solution of STT-HCPP. The Greedy algorithm that provides results quickly is among the most used heuristic algorithms. While the ACO System, the ACO which was proposed by Dorigo et al. (2007) for the first time has been inspired by the natural behavior of ants for finding food. ACO is a method that is widely utilized in recent years to address several design issues, such as routing. On the other side, the current meta-heuristic method introduced in this research is rather a different dimension to the ACO, although it is built on it. Both of the methods proposed in the research were not used for the HCPP solution in previous studies. In this study, the STT-HCPP addressed, and the algorithms used as an HCPP solution method are targeted at being the first in the literature.

\subsection{Main Steps of the Proposed Heuristic Algorithm}

The heuristic algorithm built within the framework of this study is based on a GSA algorithm and its logic can be stated as follows: by complying with the hierarchies, the list of roads that are not yet traveled will be recorded beginning from the starting node for the vehicle. This process occurs at two levels:

1. If there is an element in this list, the vehicle selects the closest road with minimal duration/distance between those roads which were not traveled before. To understand whether the arc selected in the algorithm has already been used, a matrix was generated that contains the visit information. If the value of an arc in the visit data is 0 , it means that this arc has not been used, but if the value in the visit data is 1 or more, it means that the mentioned arc has been used before. Instant location updates are provided depending on the overall distance/time value and the node chosen.

2. However, if there is no road which is not previously chosen, in other words if all roads have been used, the shortest duration would be chosen among all selected roads. After this selection, the route, distance, and visit data updates are being performed again like in the previous level.

In either of these two levels, a node or arc will be selected. The selected node is then added to the route, and the selected arc's cost value is applied to the overall cost value. Besides, an update is provided which shows that the selected arc is being used. Some controls are performed after reaching the new location. Firstly, if the algorithm has traveled all the arcs within all hierarchies and if its position is 1, we end the study. In other terms, where there is no path remaining on the network for all hierarchies and the vehicle returns from the current point to the starting point. Algorithm 1 presents the pseudo-code of the algorithm built using the programming language Matlab-2019b. The flow scheme concerning the developed algorithm is provided in Fig. 2. 


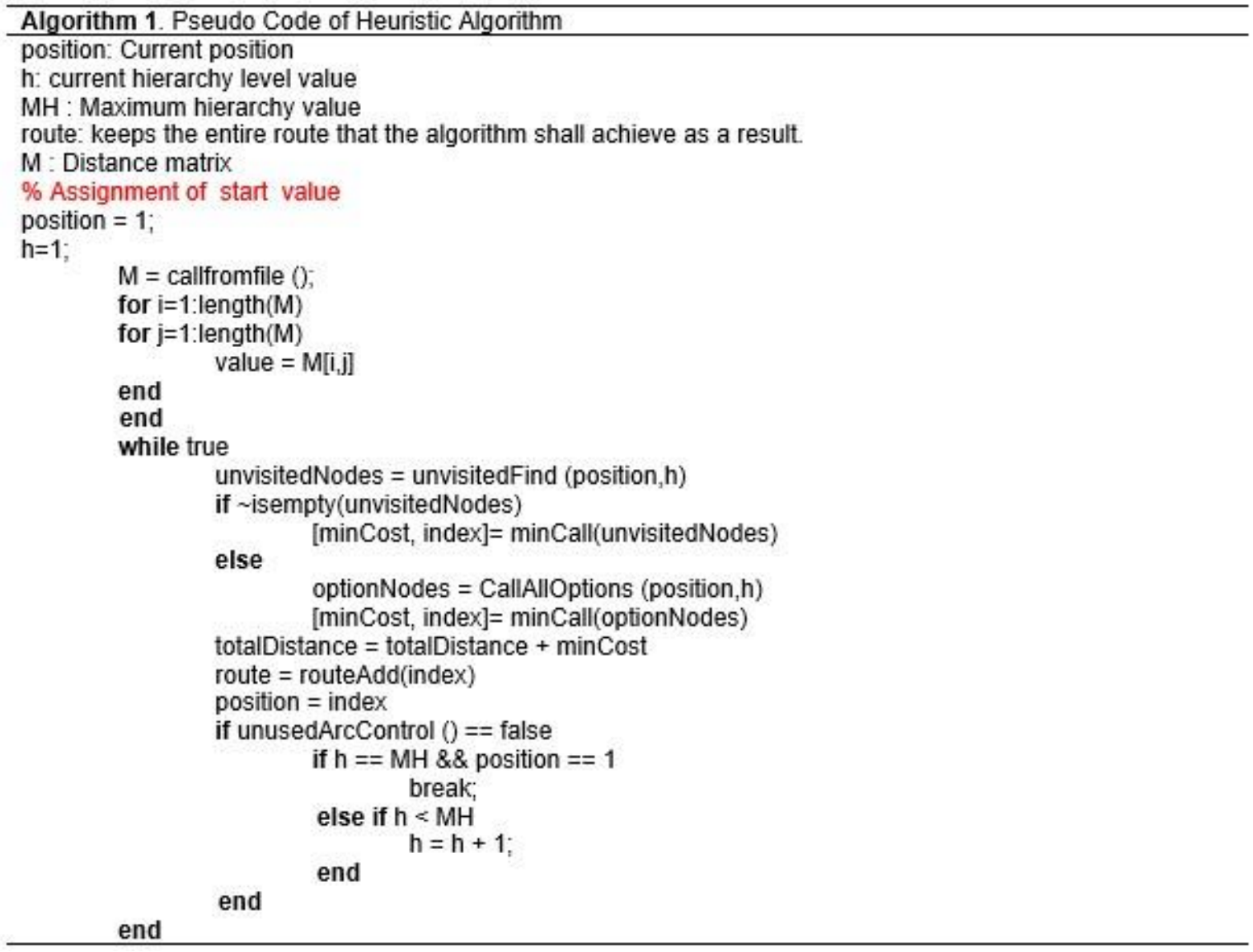




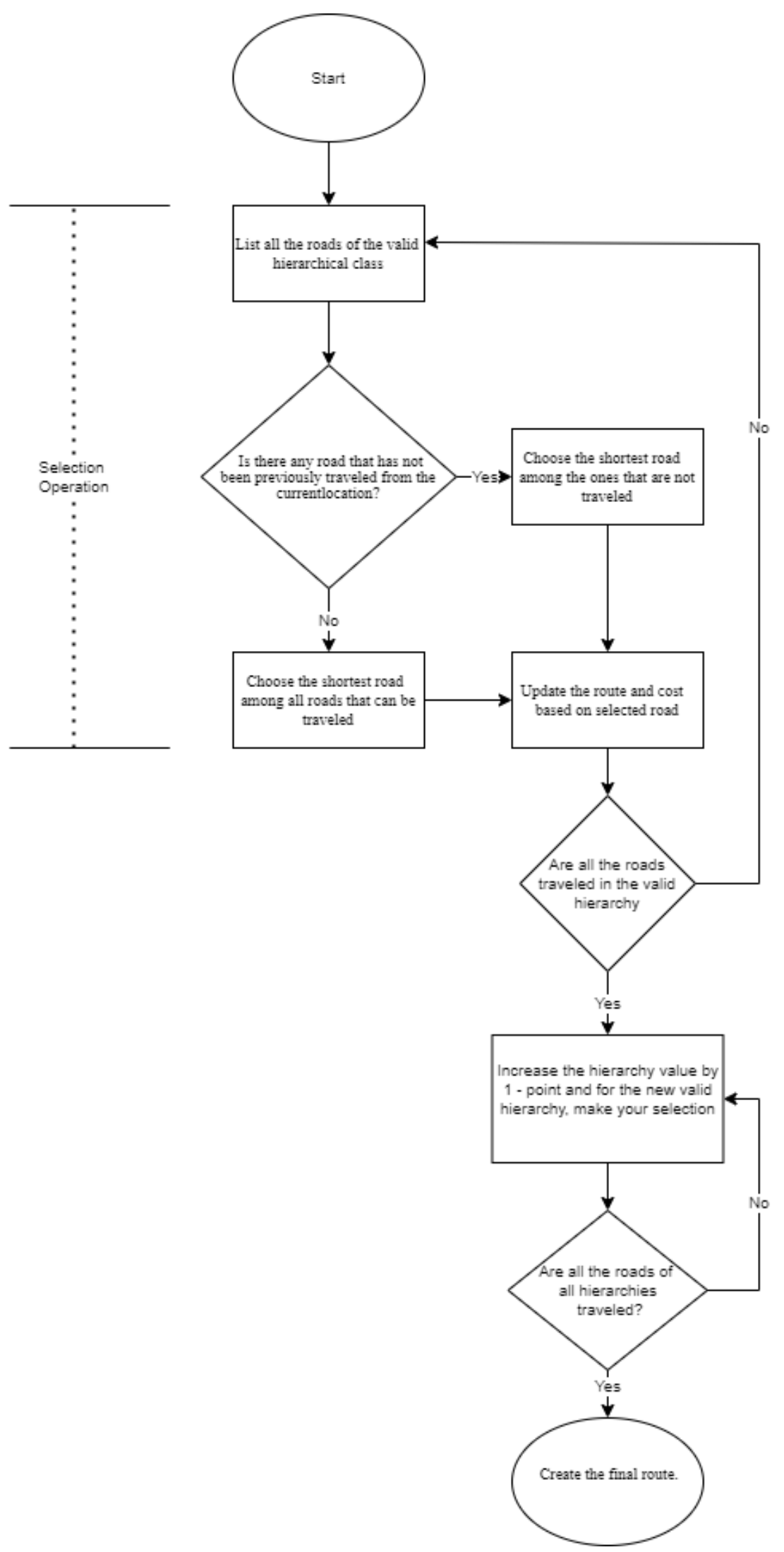

Fig. 2: Flow chart of the heuristic algorithm 


\subsection{Main Steps of the Proposed Meta-Heuristic Algorithm}

In this study, HCPP with Stochastic Travel Times (STT-HCPP), which is a new problem type, is taken into consideration. While the logic of the algorithm built is similar to the ACO, it has quite different facets to it. The proposed algorithm was coded using the Matlab-2019b programming language.

Initial State: There are three parameters in the study which should be defined firstly:

- Number of Ants

- Number of iterations

- Ant data table size

The number of ants is one of the important parameters. More ants can deliver better outcomes and enhance the approach by increasing the chance. However, providing more ants raises the number of iterations, particularly in large-scale problems, causing the working period to be extended. In the algorithm, the number of ants is determined as 300 .

The number of iterations is the number of steps taken to obtain the optimum result. It may vary depending on the problem. The low number of iterations might not be sufficient to achieve the outcome, but the high number of iterations often raises the calculation difficulties and thus, the working time (Kapukaya 2019). In the suggested algorithm, the number of iterations is determined as 1000.

Besides, there is a visit matrix for each ant in the algorithm. This matrix of the visit indicates how many occasions the ant used a path on the routes it was traveling along. The size of this visit matrix is stated as the Number of Nodes x Number of Nodes. The initial values of this visit matrix for all ants are 0. For instance, if at the start the ant moves from node $\mathrm{x}$ to node $\mathrm{y}$, the value of that path in the visit matrix is updated by increasing 1 point. The general aim is to maintain these numbers in related visit data as low. As when we use these paths less, we will have the chance to keep the length of the route shorter. Unlike TSP, this new algorithm built for HCPP doesn't depend on the amount of ant pheromone. In this type of problem, there is a possibility to cross a path more than one time. If each path was used just once, then each path may be allocated with an amount of pheromone. Although a path may be used more than once, however, the volume of pheromone here is losing its significance. Thus, visit details indicating the number of times a path was used in this method, instead of the amount of pheromone. In short, since work on the pheromone in this established ACO-based algorithm cannot be performed, the visit data is used to minimize the cost. This matrix was generated with the rationale of seeking to persuade the ant to use a path less, since the less it is used, the lower the overall cost become. If visit data were not included in the algorithm, the ant would adhere continuously to the minimum local and travel around certain locations. This problem was addressed with the visit matrix, which is used not to be bound to the local minimum, but to explore alternative options and establish the potential to produce better outcomes. Therefore, the usage aim of the algorithm's visit data can be described as follows:

1. Reducing the cost

2. To prevent traveling at a constant local minimum.

There are two different route values of ants in the algorithm. The first of these is the general route. The general route is a path value that records the nodes the ant has visited beginning from the first node and following the rules of the hierarchy until it returns to the first node again. Another route value is the one in which the route of each hierarchy is recorded one by one.

Step 1.: In the first iteration, each of the ants fills its visit matrix with "random choices, although it is likely to pursue the nearest node or the shortest path"

After the number of ants and iterations, the third parameter to be defined is the size of the ant data table. After each ant completes its route, the data is recorded in this data table, if necessary.

Two columns for each hierarchy are provided in the data table $\left(1\right.$ st hierarchy route in the $1^{\text {st }}$ column, 
while in the $2^{\text {nd }}$ column, $1^{\text {st }}$ hierarchy route length is provided). In this way, the number of columns would be 2 times of hierarchy number. In the variable named "route capacity," the value of rows in the table or how many ants' data would be stored is established. This value can be increased as desired. But the application slows down parallel to the value size. Up to 200 or 300 nodes, the ant data calculated as $3 *$ node, number can be kept.

In the initial state, all data values were zero in the visit matrix since no location was visited. After the ant completes the whole path, a matrix is generated which is called "temporary visit data."

Since there are 300 ants, there will be 300 matrices. These matrices are summed up with the mathematical logic and each value is divided by the number of ants to calculate the mean value for each path. With the equation below, in the temporary visit data matrix, we gathered the values for each ant.

temporary visit data $=$ temporary visit data $+k$ th visit data;

The mean value of these 300 matrices is determined and reported at the end of the first iteration, as it is and only applicable for the 1st iteration. Since we don't have any other details on the visit because ants have not traveled them before. In other iterations, a ratio including the amount of evaporation or the coefficient of forgetting has been used. At this phase, $80 \%$ of the old mean data is taken and the $20 \%$ becomes evaporated, and the newest one is summed up with the mean value of the ants of that iteration and the mean value is measured. Therefore, both the past visit data were used, and the mean of the visit data obtained by the ant in this iteration was summed up and as a result, the mean of the values obtained determined.

In this way; this data is also included in the arc data's third element, which belongs to a 3-dimensional matrix, the first element being hierarchy values and the second element being distance/time values. With this step, we move to the $2^{\text {nd }}$ iteration.

Step 2. (Selection process of the ant): The ant conducts the selection process in three different ways which are by looking at the routes in the database, looking at a single route, or looking at the visit data.

The Ant will start from the $1^{\text {st }}$ node and will conduct step by step the procedures mentioned below. All of those procedures mentioned are repeated on every node in the same way. In a sense, a copy of the route database matrix is transmitted to the ant before beginning operations, which keeps all the good routes in the past.

1. Before making a selection when in the $1^{\text {st }}$ or any node, it is initially tested at this moment if there are any other arcs in the hierarchy that have not been visited. If there is no arc, we move on without doing something, in other words, if all the paths in that hierarchy have been traveled, this time, the level of the ant's arc, namely the level of the hierarchy it will traverse, would be increased by 1 point.

With this procedure, it is checked whether there is an arc that has not been traveled yet. If the ant is already in the max hierarchy and the arrived node is the first one, the ant has found a route and the makes an exit. If not, the arc level is increased by 1 point and the ant moves on to the next hierarchy. Furthermore, if the ant is in the max hierarchy and has not yet reached the first node even if it has traveled all the arcs, it can proceed to travel without doing anything as per the methods described below.

2. If it continues, it implies it also has to be navigated within the framework of the graph. Now it's verified if there are any not visited arcs, on the condition that they're in the same hierarchy. If any arcs are meeting this condition, only their list is recorded. In other words, if 1-3 arcs have been used before, this arc will not be included in the list. Only the list of unused arcs is included.

3. In the $3^{\text {rd }}$ step, firstly, it is checked whether the ant is on the "new route" or not.

Selection by the database:

The "options" matrix is created for the possible targets that can be present in the second step. This matrix consists of 3 rows and $n$ number of columns. The value $n$ is the number of targets that are present in the second step. In the rows of the options matrix, 
- in the first row, the possible targets that are present in the second step are provided,

- in the second row, the mean values of pheromones are stated,

- while in the third row, probability values are presented.

In this selection, the calculation of pheromones is performed as follows. The one with the best value (the lowest) among the shortest distance/time paths is selected from the node where the ant is placed, and divided into a value such as 10000 . The purpose of this step is to determine a value as the probability value. When the same procedure is finished with all the targets it can travel, the probability of choosing the path would rise with the higher value mentioned above.

But a difficult circumstance can emerge here. To clarify, if a node within targets does not have an equivalent in the database, the mean value of its pheromone will be 0 , and choosing this node becomes impossible. However, to avoid this situation, a procedure is taken in the algorithm. With this process, by assigning the 0,1 of the smallest, in other words, the worst mean in the options matrix, a probability of being selected is provided to it even though it is certainly limited. In short, the probability of being selected by this procedure becomes not impossible anymore but has a very slim chance. With this method, while the randomness is provided at the same time, however, if it is not included in the table, we assume that it is probably not a very good route, and the probability of being selected is reduced by multiplication with 0.1 .

Also, if none of the targets have a counterpart in the database, the mean score of the pheromone of all targets will be 0 . Besides, here, all mean pheromone values are fixed to 1 and since no routes in the database would be accurate in the future, the value of the ant's "I'm on the new route" is set as true. This means that from now on, we will not be searching at the routes in the database while establishing objectives until you move on to the next hierarchy. Instead, the procedure will be taken by considering the visit data.

Hereinafter, we can discuss the pheromone effect and distance effect parameters. As mentioned earlier, what is called the pheromone effect in this algorithm is essentially the effect of visit data, which is logically the contrary to the pheromone effect in the ACO. However, it is called in the algorithm as a pheromone effect, just for the sake of clarity. The distance effect is the parameter that decides how the distance value would influence the likelihood of selecting the arc. Within the scope of the study, these values were regarded as Eq. (21) and probability values are calculated using Eq. (22).

pheromone Effect $=4$; distance Effect $=0.3$

$\left(\frac{1}{\text { distance }}\right)^{\text {distanceEffect }}+\left(\frac{1}{\text { pheromoneM }}\right)^{\text {pheromoneEffect }}=$ probalityValue

Then the probability values are determined and the $3^{\text {rd }}$ row of the matrix options are generated as well. Those probability values are normalized by leveling down to a value between $0-1$. Then they are put on the roulette table when determining the value to be chosen, and a random number between $0-1$ is obtained. This number is measured in terms of scale with the probability value, and the selection procedure is created accordingly.

After the node is selected, a different control mechanism is conducted. This control is about knowing whether there is another route in the database which matches the route generated after the target node has been selected. More clearly, for example, if node 7 is selected after node 1, the resulting route is 1-7. It should be examined whether the database includes any routes starting with 1-7. The reason for that it could have selected a path that has no route in the database. There are 3 situations to address this:

1. If there is no such route, it means the ant is on the new route now. In other terms, the database contains no other path that is appropriate for the ant's route. So now it will have to behave according to the visit data.

2. If there is only one route beginning with 1-7 in the database, the ant's "one route" function will be triggered and the ant will choose its new targets using the method discussed in the pages below.

3. If there is more than one route in the database starting with 1-7, the routes would be calculated as similar to the ant's previous preferences so far. Hence, the determination with the above logic will continue.

There is no situation that needs an extra explanation for the third case. As long as the database holds 
more than one record, calculations will continue, and a new target will be set. By proceeding in this manner, eventually at some point, there will be only one route or no route will remain. If there is just one route remaining, the ant's "one route" function is enabled, and the only path remaining is transferred to the ant from that database. After that, there will be no need to look at the database until you proceed to a new hierarchy.

\section{Selection by single route:}

If the ant follows this last remaining route, the probability to deviate from the route is modified. The brief checking takes place as accordingly; "Should I select the next target on this one remaining route or should I quit this route?"

The probability of deviating from the route is determined as 0,003 . A random number between the first node and the remaining number of nodes is determined up to 2 times the value of the remaining number of nodes in the test problems addressed within the scope of the research. For example, if there are 150 nodes left, a value between 1-300 can be generated. This method aims to try to deviate as much as possible from a location to complete the route, that is to say, at least to follow a different path when the end of the route is attained. In this way;

1. If the value generated is less than or equivalent to 3, the target node is chosen in that single route as well as the single route is discarded. If abandoned, we will act with the visit data as we no longer have a database or route.

2. If the generated value is greater than 3 , the only remaining route will be continued. Control is being performed by aiming the target and generating a random value again.

\section{Selection based on visit data}

Now we'll look at the visit details as we get to the next method of selection. Yet again, as stated before, if there are nodes not chosen before and if all the nodes that the ant might go to have already been traveled, both of their list would appear in the matrix named "options". In other terms, if there is even a single arc that was not previously used in that node, the arc is chosen without taking into consideration the arcs traveled before. No other negligence can be observed in probability calculations. But if there's more than one unused arc, a selection is created among them this time. The previously visited arcs are not included in the process. Thus, irrespective of the selection process, the matrix of the target options to which the ant can go is established in this way. The 4 criteria listed below are used as per the visit data in the selection phase:

Alpha: It is the effect of the result pheromone (visit data) on the selection and its value regarded as 0,7.

Beta: It is the coefficient that determines the effect of the arc length or cost on selection, and its value was calculated as 0,3 .

Theta: The effect of the global pheromone (visit data) on the selection was regarded as 0,6.

Gamma: The effect value of the local pheromone (visit data) on the selection was regarded as 0,4.

There are two different visit data used here. The first of these, the global visit data, is the mutual matrix where the mean score of visit data of all ants is calculated and a certain amount of it becomes evaporated. The amount of local pheromone or visit data reflects the visit data obtained up to now by the current ant. Therefore, the ant focuses at both the number of instances itself used this path previously and how much it was used by other ants on average when deciding in this manner.

The resulting amount of pheromone is the value to be derived from a calculation on the global and local visit data.

The values calculated within the study scope are not of considerable significance, and their total is 1 because they are probabilities. It is essential that only theta should be selected as 0.6 , due to the fact that we seek to draw the best value to the global visit data. And the ant depends mostly on the memory of ants who completed their route.

The following 4 cases can be identified while traveling from one node to another, depending on the visit data: 
Case-1: The arc whose probability of use would be determined was never used before. This situation is observed only in the first iteration of the application. In other terms, there will no value recorded in the global visit data concerning this arc, or the local visit data of the ant itself. The distance value is taken into account in this case, and the probability value is determined as follows.

value $=\left((1 / \text { distance })^{\wedge}\right.$ beta $)$

Case-2: $\quad$ Until now, the ant has never used this arc, but the selection process can be performed using data from global visits. In this case, the local visit data may not have a value, but the value from the global visit data is used.

resulting pheromone amount $=(\text { global amount of pheromone })^{\wedge}$ theta

value $=\left((1 / \text { resulted pheromone amount })^{\wedge}\right.$ alpha $) *\left((1 / \text { distance })^{\wedge}\right.$ beta $)$

Case-3: The ant used this arc before, therefore, local visit data, as well as data of global visits, are accessible. In such a case, when calculating the corresponding amount of pheromone, both local visit data and global visit data are utilized.

resulting pheromone amount $=\left(\right.$ local amount of pheromone ${ }^{\wedge}$ gamma $) *$ (global amount of pheromone ${ }^{\wedge}$ theta);

value $=\left((1 / \text { resulted pheromone amount })^{\wedge}\right.$ alpha $) *\left((1 / \text { distance })^{\wedge}\right.$ beta

Case-4: In the last case, the ant has used this arc before, but there is no global visit data. We observe this case only in the first iteration. And thus, the probability that an arc wasn't visited before just occurs in the first iteration. Since all arcs will be used in other iterations, there will always be global visit data. If such a case is observed during the first iteration, the selection value is determined by focusing only on the data on the local visit.

resulting pheromone amount $=$ local amount of pheromone ${ }^{\wedge}$ gamma;

value $=\left((1 / \text { resulted pheromone amount })^{\wedge}\right.$ alpha $) *\left((1 / \text { distance })^{\wedge}\right.$ beta $)$;

By evaluating the node choices from where the ant is positioned, according to the cases described above, the arc states of these nodes are determined with the variable named "value". The probability of being chosen is then determined using these values and a random number between $0-1$ is created by putting certain probability values on the roulette table using the same logic described in the previous sections. This probability value is taken into consideration and controlled and the selection is performed.

This is how the selection process ends. The node is chosen in three different ways which are by looking at the routes in the database, looking at a single route, or looking at the visit data.

Step 3. (Updates and ending): No matter how the node is chosen here, the following 3 procedures are applied:

Procedure-1: The local visit data relating to the arc used by the ant (the matrix of the ant's visit) is adjusted. The existing value is increased by 1 point to show that this arc has been used.

Procedure-2: The overall distance to the ant travels and the present hierarchy road cost values are revised. The arc distance values which are used by the ant are added.

Procedure-3: The selected node is added to the ant's route.

The ant's hierarchical route information passes through the following steps respectively. New information is added to the values which carry the best hierarchical routes in the matrix.

- Firstly, it is checked whether the capacity of this table is full or not. In this way, how many routes the table will contain (the number of rows it would have) is determined in the algorithm by creating a variable called "route capacity". If the route capacity has not been met yet, the ant's hierarchical route data are respectively incorporated as it is.

- If this route capacity is fulfilled, each hierarchical route of the ant is compared with the route costs 
that are listed in the table at the columns of that hierarchy. If it is better than any cost value, the route and cost value of the hierarchy we control of that ant is added instead of the worst (highest cost) route in the table. Then it proceeds to the other hierarchy route and that is checked as well. In this way, all hierarchy routes can be controlled.

With these procedures, the aim is enabling the table to maintain the best of each route of hierarchy. These procedures are repeated after each ant completes its route. The iteration ends after all the ants finish their routes. At the end of the iteration, visit data are collected and updated. These procedures are carried out in the same way as defined in the preceding sections.

The algorithm's pseudo code developed within the study scope and its detailed steps are shown in Algorithm 2. 


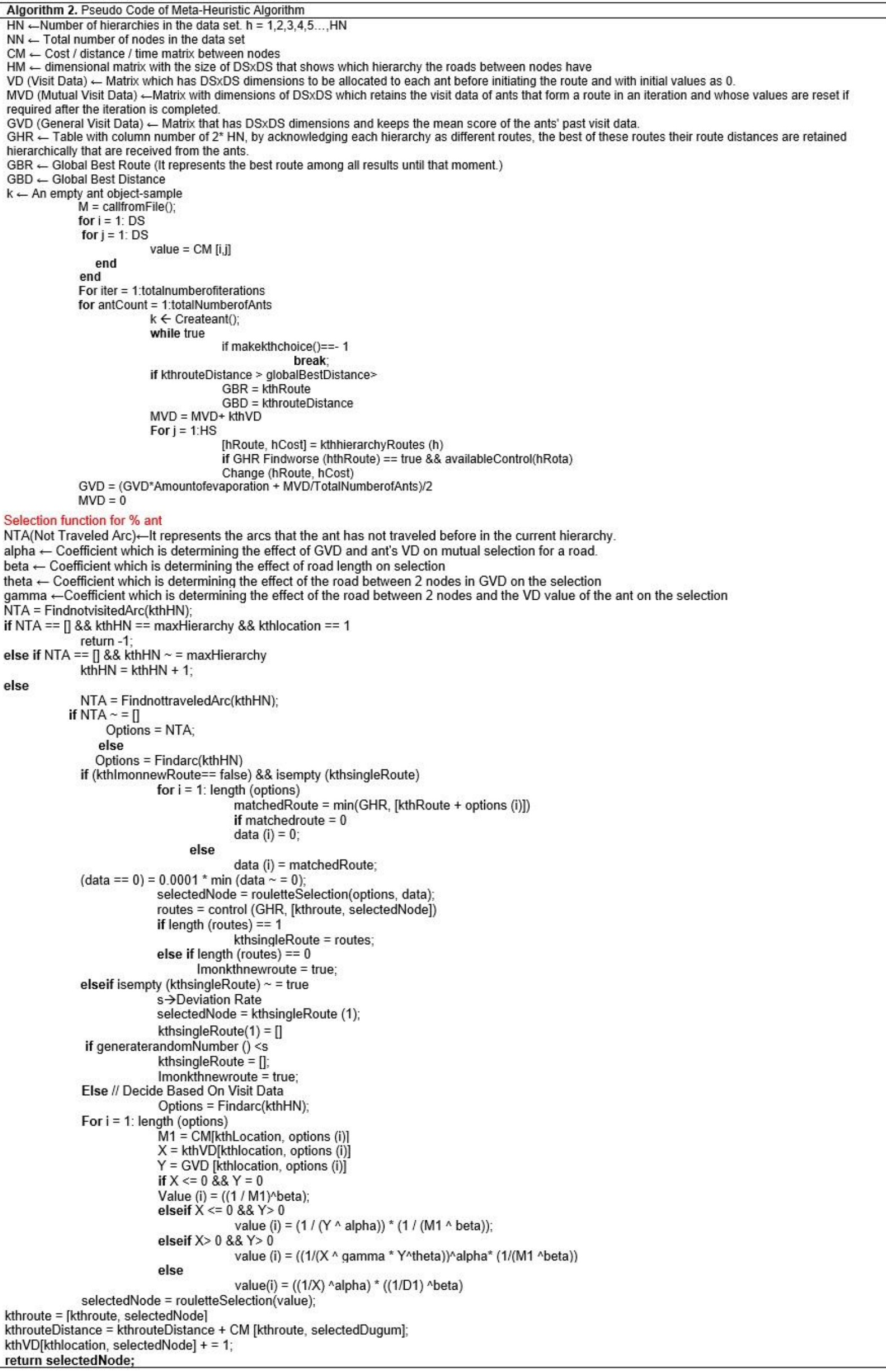


Last but not least, distance values have been used directly for the HCPP solution in both algorithms in the objective function, but instead of the distance values for STT-HCPP, durations with defined mean and standard deviation values, and which showed normal distribution characteristics have been used. Thus, the "random function" that generates random values for time values is applied in the algorithms.

\section{Results}

A sufficient amount of test problems is needed to test the heuristic and meta-heuristic algorithms of the STT-HCPP mathematical model which is presented in this study. In the literature regarding HCPP in have two small-sized test samples. Within the framework of this study, randomly generated test samples mentioned in the studies of Çodur and Yilmaz (2020) have been used.

Considering a $G=(V, E)$ graph, the test problems are stated as the set of nodes $V=\{10,20,30,40,50\}$ and the set of hierarchies $H=\{2,3,4,5\}$. To assess the performance of the Cplex solver, the GSA-based heuristic and the ACO-based meta-heuristic proposed comparatively, within the scope of the research, small-scale test problems were regarded as HCPP by considering the distances. Those examples were later modified to random travel times with normal distribution rather than the distances that are the study's main subject. In these test problems, the distance values were transformed into time values based on the city's legal speed limit. In algorithms, random time matrices were derived from defined mean and standard deviation values, and by using the suggested methods, solutions were pursued test problems.

First, optimum findings were achieved with the Cplex solver for small size problems by using HCPP mathematical model. These problems are the 13 and 18- edged test problems with 2,3,4, and 5 hierarchies while having 10 nodes. The maximum time to solve the aforementioned problems is determined as 1 hour (3600 seconds). Then the performance of the Cplex solver, the proposed GSA-based heuristic, and the ACO-based meta-heuristic was assessed comparatively with these results. Table 2 presents the results obtained from the Cplex solver which belongs to the 10 node test problems. In the first column of the table, the name of the test problem, in the second column, the hierarchy number of the problem (h), in the third column the number of nodes of the problem $(|\mathrm{V}|)$, and finally in the fourth column the information about the problem's edge no $(|\mathrm{E}|)$ are provided. In the fifth, sixth, and seventh columns; information is provided about the cluster of paths divided into hierarchical classes of the problem, the optimal route found using the Cplex solver, and the optimal route distance, respectively.

Table 2 Results obtained with the HCPP mathematical model 


\begin{tabular}{|c|c|c|c|c|c|c|}
\hline $\begin{array}{l}\text { Test } \\
\text { Problem } \\
\text { name }\end{array}$ & $\boldsymbol{h}$ & $|\mathbf{V}|$ & $|\mathbf{E}|$ & $\begin{array}{c}\text { The Cluster of Roads Divided into } \\
\text { Hierarchical Classes }\end{array}$ & Optimum Route & $\begin{array}{l}\text { Optimum } \\
\text { Tour } \\
\text { Distance }\end{array}$ \\
\hline Rnd_inst25 & 2 & 10 & 13 & $\begin{array}{l}E_{1}(i, j) / 1.5,2.3,2.5,2.6,3.9,4.9,5.7 / \\
E_{2}(i, j) / 1.6,3.10,4.8,6.10,7.8,8.10 /\end{array}$ & $\begin{array}{l}1-5-7-5-2-6-2-3-9- \\
4-8-7-8-10-3-10- \\
6-1\end{array}$ & 1281 \\
\hline Rnd_inst26 & 2 & 10 & 18 & $\begin{array}{l}E_{1}(i, j) / 1.2,1.5,1.9,2.5,2.7,2.8,3.8 \\
3.9,4.5,4.6,4.8,4.9,7.9 / \\
E_{2}(i, j) / 1.6,1.8,5.10,6.9,9.10 /\end{array}$ & $\begin{array}{l}1-2-8-4-8-3-9-4-6- \\
4-5-2-7-9-1-5-10- \\
9-6-1-8-1\end{array}$ & 1417 \\
\hline Rnd_inst27 & 3 & 10 & 13 & $\begin{array}{l}E_{l}(i, j) / 1.5,1.6,4.5,6.9 / \\
E_{2}(i, j) / 2.6,4.6,4.7,4.8,5.8 / \\
E_{3}(i, j) / 1.4,4.9,4.10,8.9 /\end{array}$ & $\begin{array}{l}1-6-9-6-1-5-4-7-4- \\
6-2-6-4-8-5-8-9-4- \\
10-4-1\end{array}$ & 1386 \\
\hline Rnd_inst28 & 3 & 10 & 18 & $\begin{array}{l}E_{l}(i, j) / 1.3,1.5,1.6,1.7,2.9,5.7,6.7, \\
6.9 / \\
E_{2}(i, j) / 2.4,4.5,4.8,4.9,4.10,5.8,8.9 / \\
E_{3}(i, j) / 3.4,3.8,3.10 /\end{array}$ & $\begin{array}{l}1-3-1-7-6-7-5-1-6- \\
9-2-4-8-9-4-10-4- \\
5-8-3-4-10-3-1\end{array}$ & 1516 \\
\hline Rnd_inst29 & 4 & 10 & 13 & $\begin{array}{l}E_{1}(i, j) / 1.8,1.9,8.9 / \\
E_{2}(i, j) / 1.2,1.4,2.5,2.6,2.7,4.7 / \\
E_{3}(i, j) / 1.6,1.10 / \\
E_{4}(i, j) / 7.9,9.10 /\end{array}$ & $\begin{array}{l}1-9-8-1-2-5-2-7-4- \\
1-2-6-1-10-9-7-4- \\
1\end{array}$ & 1249 \\
\hline Rnd_inst30 & 4 & 10 & 18 & $\begin{array}{l}E_{l}(i, j) / 1.2,1.7,2.5,3.7,5.7 / \\
E_{2}(i, j) / 1.4,1.10,2.10,4.7,7.10,9.10 / \\
E_{3}(i, j) / 4.5,4.10,5.6,6.10 / \\
E_{4}(i, j) / 6.7,7.8,8.9 /\end{array}$ & $\begin{array}{l}1-7-3-7-5-2-1-10- \\
9-10-7-4-1-2-10- \\
4-5-6-10-9-8-7-6- \\
7-1\end{array}$ & 1582 \\
\hline Rnd_inst31 & 5 & 10 & 13 & $\begin{array}{l}E_{l}(i, j) / 1.2,1.7,2.6,5.10,6.10 / \\
E_{2}(i, j) / 5.7,6.7 / \\
E_{3}(i, j) / 5.9,6.9 / \\
E_{4}(i, j) / 2.4,4.5 / \\
E_{5}(i, j) / 1.3,3.5 /\end{array}$ & $\begin{array}{l}1-7-1-2-6-10-5-7- \\
6-9-5-4-2-4-5-3-1\end{array}$ & 1013 \\
\hline Rnd_inst32 & 5 & 10 & 18 & $\begin{array}{l}E_{l}(i, j) / 1.4,1.5,1.6,4.6,6.8,6.9 / \\
E_{2}(i, j) / 2.4,3.4,3.6,3.10,4.10 / \\
E_{3}(i, j) / 2.3,2.8,2.10 / \\
E_{4}(i, j) / 5.6,6.10 / \\
E_{5}(i, j) / 3.5,3.7 /\end{array}$ & $\begin{array}{l}1-4-6-8-6-1-5-1-6- \\
9-6-3-10-4-2-4-3- \\
2-8-2-10-6-5-3-7- \\
3-4-1\end{array}$ & 2006 \\
\hline
\end{tabular}

The results that comparatively assess the performance of the Cplex solver, heuristic, and meta-heuristic approaches are shown in Table 3. In the problem solution, the results were obtained by using the Matlab$2019 \mathrm{~b}$ program and a computer with 32 GB of memory and an Intel Core i9 $9900 \mathrm{~K}$ processor. In the first column of this table, the name of the test problem is given while in the second, third, and fourth columns, the number of nodes $(|\mathrm{V}|)$, the number of hierarchies $(|\mathrm{h}|)$, and the number of edges $(|\mathrm{E}|)$ of that problem are presented respectively. The fifth, sixth and seventh columns describing the conclusions drawn from the Cplex solver indicate the best objective function value identified, the time of the computer solution, and the 
percentage difference between the best solution found by the Cplex solver and the best objective function value stated by Cplex. $\left(\mathrm{O}^{\mathrm{Cplex}}, \mathrm{T}^{\mathrm{Cplex}}\right.$, Gap). While the eighth and ninth columns, showing the results obtained from the proposed ACO-based meta-heuristic approach, are providing respectively the best objective function value $\left(\mathrm{O}^{\mathrm{ACO}}\right)$ and the computer $\mathrm{CPU}$ time (seconds) of the best solution $\left(\mathrm{T}^{\mathrm{ACO}}\right)$. Similarly, the tenth and eleventh columns showing the results obtained from the proposed GSA-based heuristic approach are respectively presenting the best objective function value $\left(\mathrm{O}^{\mathrm{GSA}}\right)$ and the computer CPU time (seconds) of the best solution ( $\mathrm{T}^{\mathrm{GSA}}$ ).

The conditions for stopping the algorithms presented in this study were set as follows:

- When the optimum solution is achieved in small size problems,

- For medium and large-sized problems, when the fixed number of iterations is reached is fulfilled, the algorithm termination process is performed.

Table 3 HCPP mathematical model, heuristic, and meta-heuristic approaches solution results

\begin{tabular}{lllllllllll}
\hline $\begin{array}{l}\text { Name of the } \\
\text { test problem }\end{array}$ & $|V|$ & $|h|$ & $|E|$ & $O^{\text {Cplex }}$ & $T^{\text {Cplex }}(\mathrm{s})$ & $\begin{array}{l}\text { Gap } \\
(\%)\end{array}$ & $O^{\text {ACO }}$ & $T^{\text {ACO }}(\mathrm{s})$ & $O^{\mathrm{GSA}}$ & $T^{\mathrm{GSA}}(\mathrm{s})$ \\
\hline Rnd_inst25 & 10 & 2 & 13 & 1281 & 0,94 & 0 & 1281 & 0,05 & 2422 & 0,08 \\
Rnd_inst26 & 10 & 2 & 18 & 1417 & 483,28 & 0 & 1417 & 0,01 & 3687 & 0,01 \\
Rnd_inst27 & 10 & 3 & 13 & 1386 & 32,14 & 0 & 1386 & 0,70 & 3762 & 0,02 \\
Rnd_inst28 & 10 & 3 & 18 & 1516 & 124,25 & 0 & 1516 & 0,94 & 3226 & 0,04 \\
Rnd_inst29 & 10 & 4 & 13 & 1249 & 2,02 & 0 & 1249 & 0,03 & 3400 & 0,01 \\
Rnd_inst30 & 10 & 4 & 18 & 1582 & 65,34 & 0 & 1582 & 0,14 & 4210 & 0,03 \\
Rnd_inst31 & 10 & 5 & 13 & 1013 & 0,77 & 0 & 1013 & 0,13 & 3978 & 0,20 \\
Rnd_inst32 & 10 & 5 & 18 & 2006 & 182,53 & 0 & 2006 & 0,33 & 6397 & 0,02 \\
\hline
\end{tabular}

For all 10-node, 13 and 18-edge problems; optimum solutions and routes were obtained in seconds/minutes with the Cplex solver and the proposed meta-heuristic. It is shown that with the suggested heuristic approach, those results are close to optimal. This is an expected result. Meta-heuristics perform better results than heuristics. Furthermore, these results are provided in Fig. 3. When the computational times are evaluated, it was observed that the average score of computer CPU time used by the Cplex solver was 383 times higher than the suggested meta-heuristic, and the mean score of the computer CPU time used by the suggested meta-heuristic algorithm was 5,68 times higher than suggested heuristic. Thus, it can be said that on these test problems, these three methods are successful at certain rates. The results obtained regarding the computational times are presented in Fig. 4. 


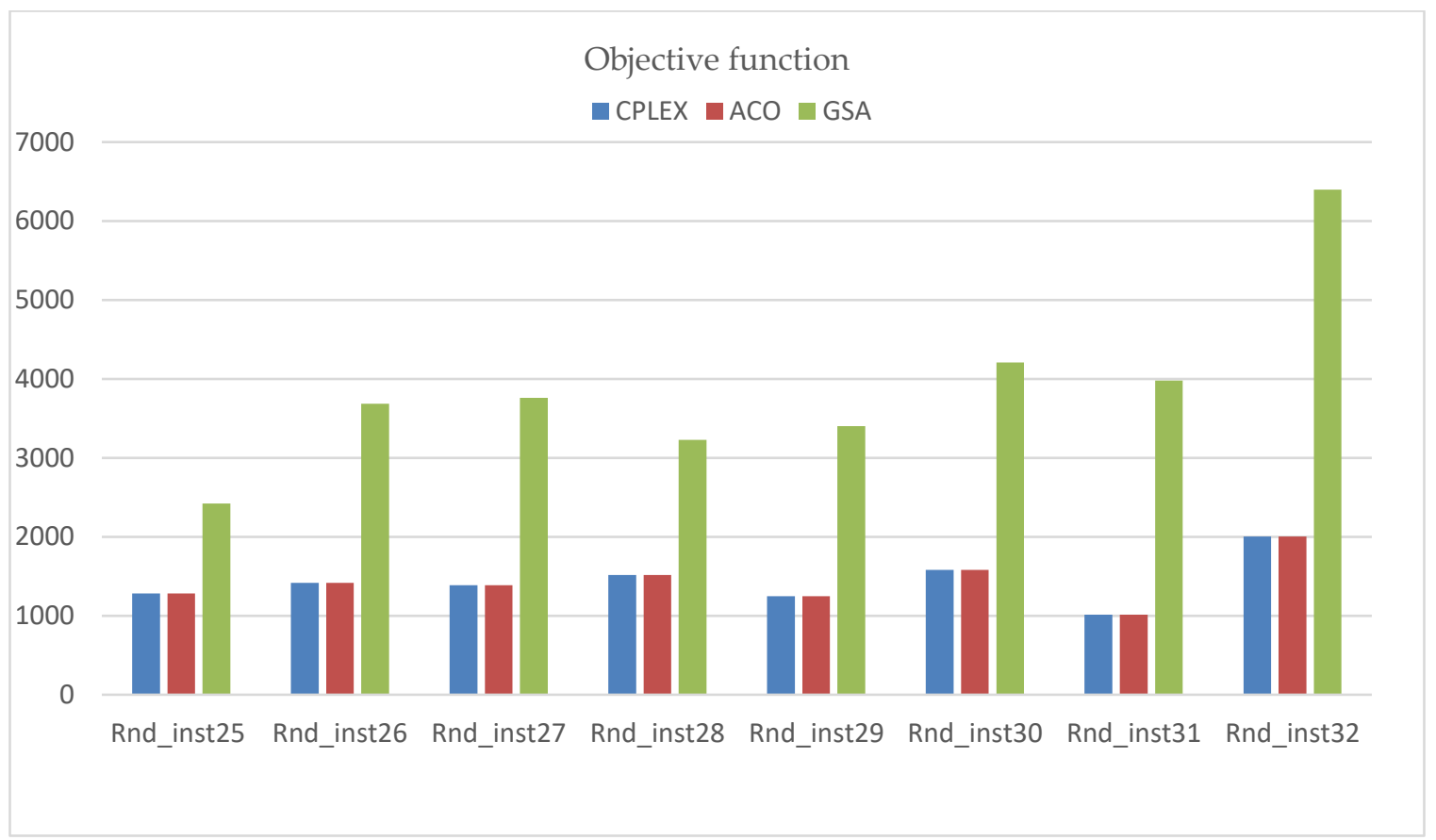

Fig. 3: The objective function values for small-size HCPP

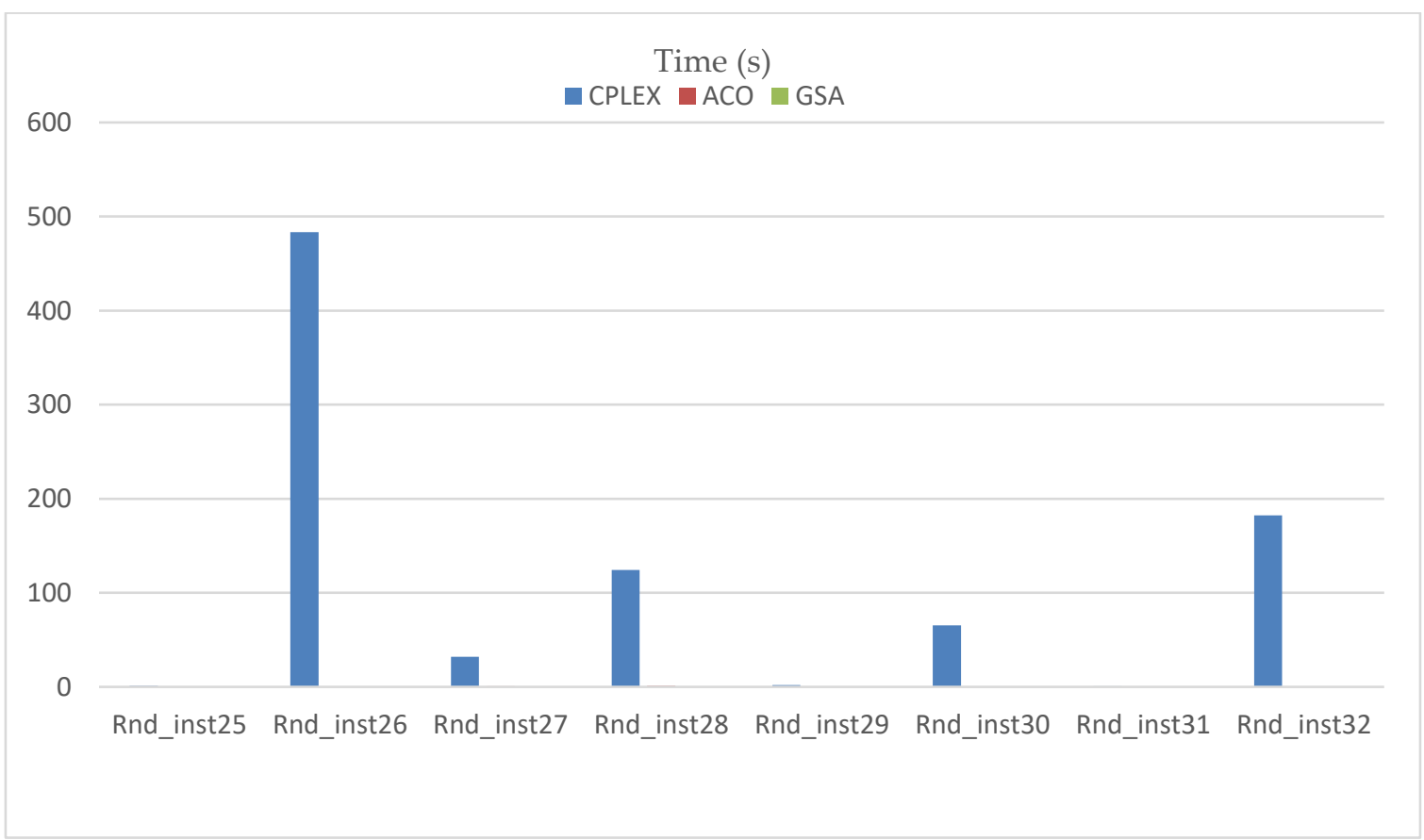

Fig. 4: Computational times for the small-size HCCP

Table 4. and Table 5 presents the results obtained for STT-HCPP by using algorithms created, and the performance of the algorithms. In the first column of this table, the name of the test problem is given while in the second, third, and fourth columns, the number of nodes $(|\mathrm{V}|)$, the number of hierarchies $(|\mathrm{h}|)$, and the number of edges $(|\mathrm{E}|)$ of that problem are presented respectively. The fifth and sixth columns are presenting respectively the best objective function value $\left(\mathrm{O}^{\mathrm{ACO}}\right)$ obtained from the proposed ACO-based meta-heuristic 
approach and the computer CPU time (seconds) of the best solution $\left(\mathrm{T}^{\mathrm{ACO}}\right)$. Similarly, the seventh and eighth columns showing the results obtained from the proposed GSA-based heuristic approach are respectively presenting the best objective function value $\left(\mathrm{O}^{\mathrm{GSA}}\right)$ and the computer CPU time (seconds) of the best solution $\left(\mathrm{T}^{\mathrm{GSA}}\right)$.

In all instances with 10 and 20 nodes ACO-based meta-heuristic approach found better objective function value than GSA-based heuristic approach. In these problems, the average best objective function value found by the GSA-based heuristic was 2.44 times higher than the average best objective function value found by the ACO-based meta-heuristic. However, it is seen that the heuristic algorithm gives better results as the number of edges increases For example, for Rnd_inst 25 with 10 nodes and 13 sides, while the value of the objective function found by the heuristic is 2.19 times higher than the value of the objective function of the meta-heuristic; for Rnd_inst.annex1 with 10 nodes and 30 sides, the value of the objective function the heuristic finds is 1.64 times higher than the value of the objective function of the meta-heuristic. Although the ACO-based meta-heuristic algorithm finds better results in all of these test instances, the average time used by the ACO-based meta-heuristic algorithm is 204 times larger than the GSA-based heuristic. However, the average time used by ACO is less than 1 minute in all of these test instances. The average time used by GSA is less than 1 second. This shows that the algorithms are performing well.

Table 4 Results of algorithms for instances with 10 and 20 nodes

\begin{tabular}{|c|c|c|c|c|c|c|c|}
\hline $\begin{array}{l}\text { Test } \\
\text { Problems }\end{array}$ & $|V|$ & $|h|$ & $|E|$ & $O^{\mathrm{ACO}}$ & $\mathrm{T}^{\mathrm{ACO}}(\mathrm{s})$ & $O^{\mathrm{GSA}}$ & $\mathrm{T}^{\mathrm{GSA}}(\mathrm{s})$ \\
\hline Rnd_inst25 & 10 & 2 & 13 & 352,93 & 0,07 & 772,21 & 0,04 \\
\hline Rnd_inst26 & 10 & 2 & 18 & 382,44 & 0,23 & 866,87 & 0,01 \\
\hline Rnd_inst.annex1 & 10 & 2 & 30 & 625,66 & 3,98 & 1023,12 & 0,01 \\
\hline Rnd_inst27 & 10 & 3 & 13 & 393,09 & 0,18 & 1137,35 & 0,03 \\
\hline Rnd_inst28 & 10 & 3 & 18 & 410,59 & 5,16 & 942,59 & 0,01 \\
\hline Rnd_inst.annex 2 & 10 & 3 & 30 & 643,76 & 5,72 & 1626,07 & 0,01 \\
\hline Rnd_inst29 & 10 & 4 & 13 & 362,71 & 0,31 & 1009,98 & 0,02 \\
\hline Rnd_inst30 & 10 & 4 & 18 & 418,11 & 0,60 & 1159,91 & 0,01 \\
\hline Rnd_inst.annex3 & 10 & 4 & 30 & 752,54 & 5,86 & 1512,15 & 0,01 \\
\hline Rnd_inst31 & 10 & 5 & 13 & 277,27 & 0,14 & 1135,04 & 0,02 \\
\hline Rnd_inst32 & 10 & 5 & 18 & 568,42 & 0,66 & 1652,14 & 0,02 \\
\hline Rnd_inst.annex4 & 10 & 5 & 30 & 758,23 & 5,40 & 2513,82 & 0,02 \\
\hline Rnd_inst33 & 20 & 2 & 55 & 1146,39 & 12,03 & 2441,45 & 0,04 \\
\hline Rnd_inst34 & 20 & 2 & 76 & 1658,10 & 1,871 & 2484,46 & 0,02 \\
\hline Rnd_inst.annex5 & 20 & 2 & 127 & 2329,00 & 12,67 & 4126,64 & 0,02 \\
\hline Rnd_inst35 & 20 & 3 & 55 & 1530,85 & 0,029 & 2259,77 & 0,02 \\
\hline Rnd_inst36 & 20 & 3 & 76 & 1674,52 & 11,49 & 3924,99 & 0,02 \\
\hline Rnd_inst.annex6 & 20 & 3 & 127 & 2466,04 & 15,15 & 5785,19 & 0,03 \\
\hline Rnd_inst37 & 20 & 4 & 55 & 1384,88 & 10,49 & 3795,34 & 0,03 \\
\hline Rnd_inst38 & 20 & 4 & 76 & 1708,00 & 11,37 & 5313,04 & 0,03 \\
\hline Rnd_inst.annex7 & 20 & 4 & 127 & 2721,70 & 15,43 & 7438,67 & 0,03 \\
\hline Rnd_inst39 & 20 & 5 & 55 & 1418,64 & 11,83 & 5436,73 & 0,12 \\
\hline Rnd_inst40 & 20 & 5 & 76 & 1821,66 & 13,48 & 4918,01 & 0,16 \\
\hline Rnd_inst.annex 8 & 20 & 5 & 127 & 2632,03 & 16,02 & 8564,58 & 0,05 \\
\hline
\end{tabular}


Table 5 Results of algorithms for instances with 30, 40 and 50 nodes

\begin{tabular}{|c|c|c|c|c|c|c|c|}
\hline $\begin{array}{l}\text { Test } \\
\text { Problems }\end{array}$ & $|V|$ & $|h|$ & $|E|$ & $O^{\mathrm{ACO}}$ & $\mathrm{T}^{\mathrm{ACO}}(\mathrm{s})$ & $O^{\mathrm{GSA}}$ & $\mathrm{T}^{\mathrm{GSA}}(\mathrm{s})$ \\
\hline Rnd_inst41 & 30 & 2 & 125 & 2722,09 & 21,26 & 4821,46 & 0,05 \\
\hline Rnd_inst42 & 30 & 2 & 174 & 3434,05 & 99,91 & 5496,91 & 0,03 \\
\hline Rnd_inst.annex9 & 30 & 2 & 290 & 5802,46 & 49,71 & 9330,78 & 0,04 \\
\hline Rnd_inst43 & 30 & 3 & 125 & 2831,39 & 20,05 & 6515,62 & 0,04 \\
\hline Rnd_inst44 & 30 & 3 & 174 & 3791,72 & 33,71 & 6951,35 & 0,03 \\
\hline Rnd_inst.annex10 & 30 & 3 & 290 & 5939,79 & 33,29 & 12939,8 & 0,04 \\
\hline Rnd_inst45 & 30 & 4 & 125 & 2888,26 & 22,19 & 8046,46 & 0,03 \\
\hline Rnd_inst46 & 30 & 4 & 174 & 4139,53 & 28,40 & 10155,04 & 0,05 \\
\hline Rnd_inst.annex11 & 30 & 4 & 290 & 6165,37 & 40,24 & 13162,52 & 0,05 \\
\hline Rnd_inst47 & 30 & 5 & 125 & 3557,33 & 24,65 & 11733,46 & 0,05 \\
\hline Rnd_inst48 & 30 & 5 & 174 & 4050,81 & 28,64 & 13744,53 & 0,04 \\
\hline Rnd_inst.annex12 & 30 & 5 & 290 & 6656,80 & 35,87 & 23174,55 & 0,07 \\
\hline Rnd_inst49 & 40 & 2 & 223 & 4555,29 & 879,91 & 20488,21 & 0,25 \\
\hline Rnd_inst50 & 40 & 2 & 312 & 6381,16 & 39,54 & 10347,59 & 0,09 \\
\hline Rnd_inst.annex13 & 40 & 2 & 520 & 10015,17 & 94,71 & 17383,74 & 0,10 \\
\hline Rnd_inst51 & 40 & 3 & 223 & 5338,09 & 35,11 & 10404,01 & 0,14 \\
\hline Rnd_inst52 & 40 & 3 & 312 & 6500,28 & 108,63 & 13902,66 & 0,11 \\
\hline Rnd_inst.annex14 & 40 & 3 & 520 & 10528,82 & 64,94 & 22885,18 & 0,12 \\
\hline Rnd_inst53 & 40 & 4 & 223 & 5537,26 & 42,68 & 15607,80 & 0,13 \\
\hline Rnd_inst54 & 40 & 4 & 312 & 7655,61 & 53,73 & 15705,82 & 0,12 \\
\hline Rnd_inst.annex15 & 40 & 4 & 520 & 10939,70 & 187,26 & 27905,17 & 0,16 \\
\hline Rnd_inst55 & 40 & 5 & 223 & 6148,42 & 41,28 & 19498,20 & 0,19 \\
\hline Rnd_inst56 & 40 & 5 & 312 & 7766,13 & 48,61 & 23646,31 & 0,17 \\
\hline Rnd_inst.annex16 & 40 & 5 & 520 & 11776,57 & 78,08 & 35088,12 & 0,19 \\
\hline Rnd_inst57 & 50 & 2 & 350 & 7322,92 & 99,26 & 10628,49 & 0,09 \\
\hline Rnd_inst58 & 50 & 2 & 490 & 9513,57 & 896,83 & 14235,58 & 0,10 \\
\hline Rnd_inst.annex17 & 50 & 2 & 817 & 15671,36 & 667,35 & 23720,74 & 0,17 \\
\hline Rnd_inst59 & 50 & 3 & 350 & 7935,79 & 119,30 & 18032,64 & 0,17 \\
\hline Rnd_inst60 & 50 & 3 & 490 & 10710,27 & 72,16 & 18779,64 & 0,15 \\
\hline Rnd_inst.annex18 & 50 & 3 & 817 & 16414,84 & 150,80 & 28182,93 & 0,16 \\
\hline Rnd_inst61 & 50 & 4 & 350 & 8669,10 & 63,84 & 23577,02 & 0,18 \\
\hline Rnd_inst62 & 50 & 4 & 490 & 11633,40 & 73,47 & 31106,80 & 0,20 \\
\hline Rnd_inst.annex19 & 50 & 4 & 817 & 17155,59 & 108,33 & 44820,77 & 0,22 \\
\hline Rnd_inst63 & 50 & 5 & 350 & 9572,20 & 73,16 & 22751,62 & 0,19 \\
\hline Rnd_inst64 & 50 & 5 & 490 & 12434,50 & 76,82 & 37097,74 & 0,22 \\
\hline Rnd_inst.annex 20 & 50 & 5 & 817 & 17939,30 & 198,57 & 47792,28 & 0,24 \\
\hline
\end{tabular}


As the problem size in STT-HCPP increases, the complexity of the network increases, and thus the time in both approaches for the solution increases. For example, network representations of Rnd_inst 25 and Rnd_inst.annex20 are represented in Fig. 5.

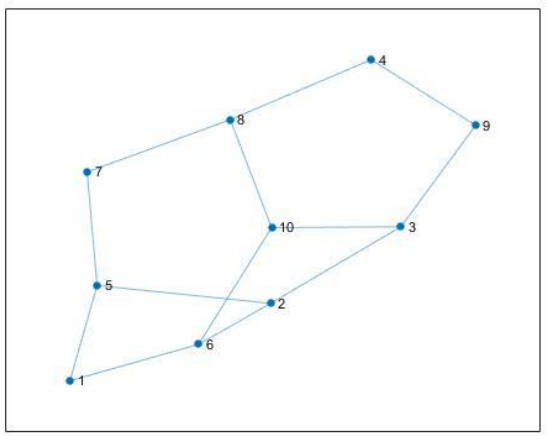

(a)

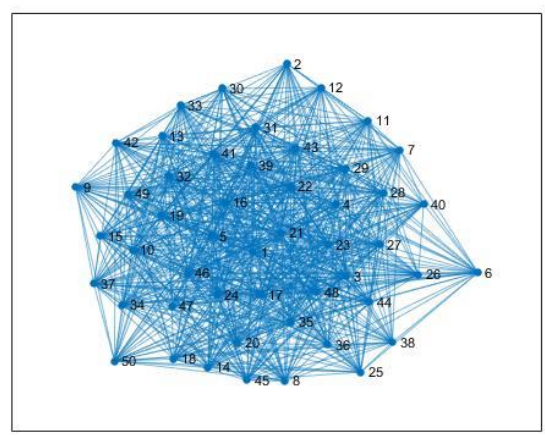

(b)

Fig. 5: Hierarchical network of instances (a) for Rnd_inst25; (b) for Rnd_inst.annex20

For the test problems discussed within the scope of the study, average objective function values, and computational times are provided in Fig.6 and Fig.7 respectively.

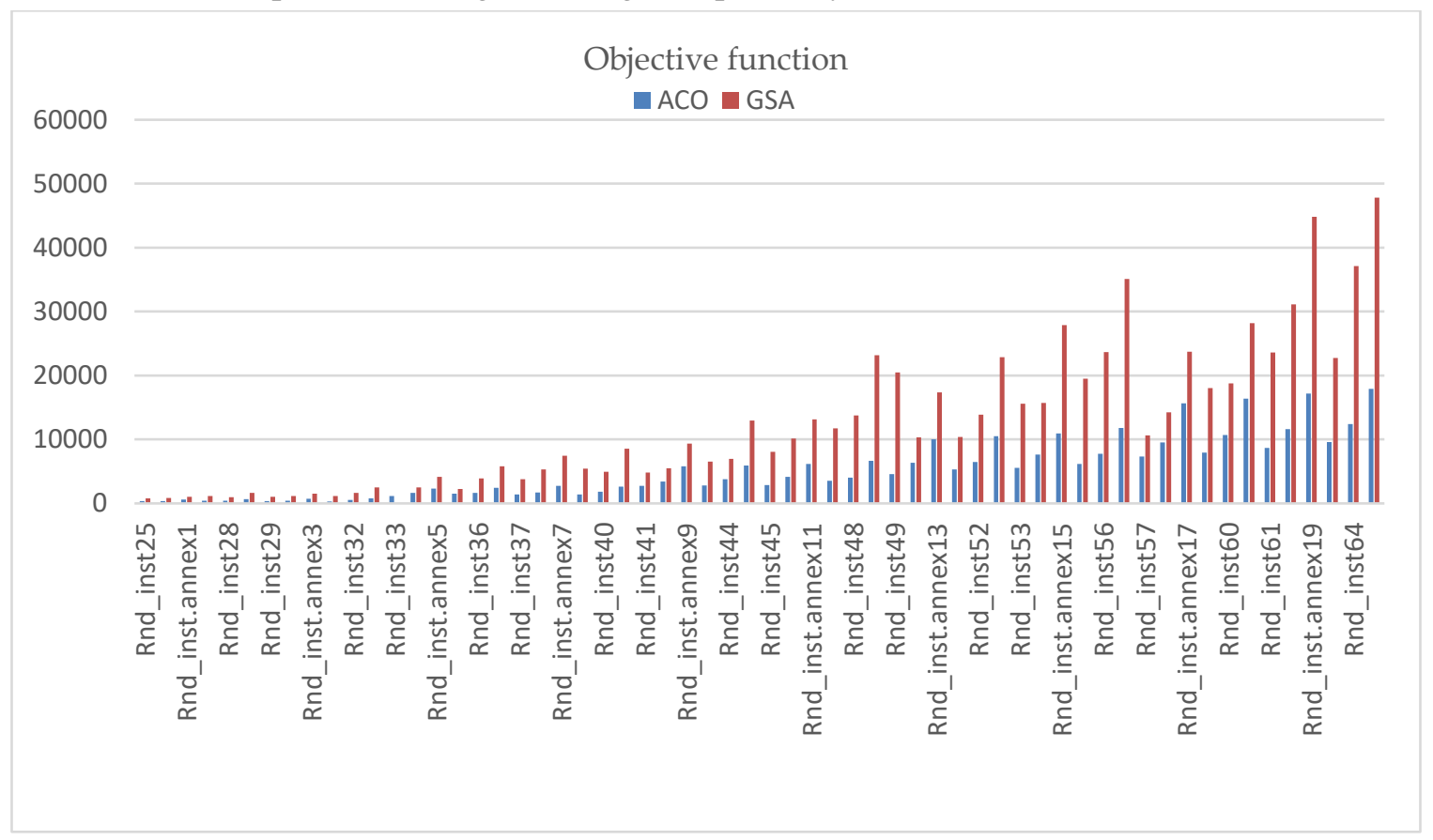

Fig. 6: The objective function of the suggested algorithms 


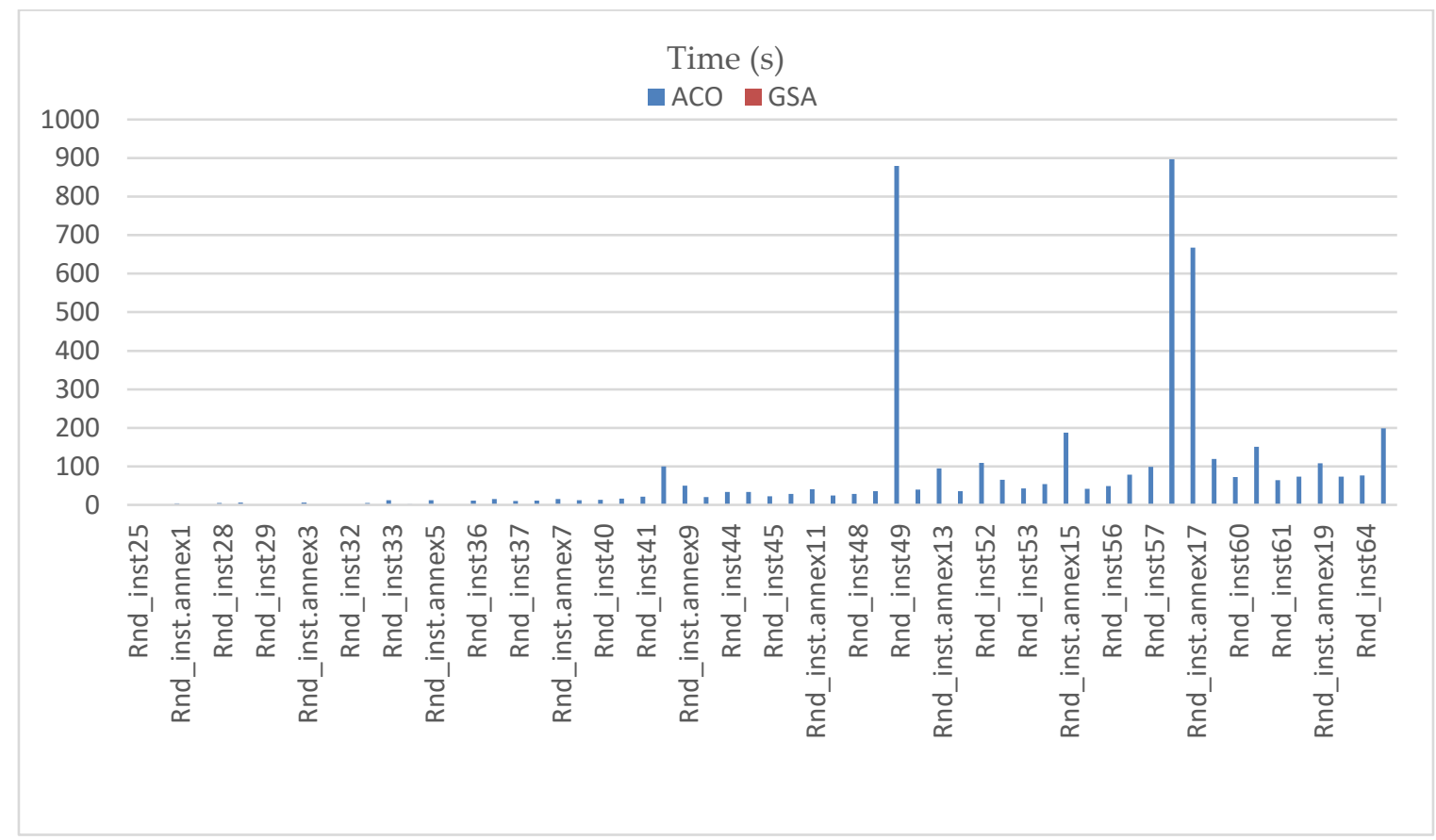

Fig. 7: Computational times for the suggested algorithms

In all instances with 30, 40 and 50 nodes, the average objective function value found by GSA-based heuristic algorithm is 2.34 times greater than the ACO-based meta-heuristic. Although the ACO-based meta-heuristic algorithm finds better results in all of these test instances, the average time used by the ACObased meta-heuristic algorithm is 1083,8 times larger than the GSA-based heuristic. In these examples, while ACO based meta-heuristic algorithm provides solutions within seconds/minutes, GSA-based heuristic's average computer time is less than 1 second.

In all of the examples covered in the study, ACO based meta-heuristic performed better than the GSAbased heuristic in terms of average objective function value. However, the average computer time spent by the GSA-based heuristic on all of these test instances is less than ACO-based meta-heuristic. As a consequence, one of the ACO's main advantages is that they undertake an intense and profound search in the solution space. On the other hand, the established heuristic algorithm provided worse results as it was searching only locally. Even though greedy algorithms do not always provide the best solution for various types of problems, they do find the most suitable solution to some problems.

\section{Discussion}

In this study, a new problem type namely the STT-HCPP is presented. In many other HCPP studies in the literature, attention was given to the distance traveled by the vehicles throughout the routing. That being said, in today's modern world in which the competition in the market is at its height, identifying the travel times between nodes is necessary in order not to waste time and execute the planning process accurately. In most real-life issues, due to unpredictable weather conditions, environmental factors, or changing traffic density, it is not always possible to express the travel times and speed with exact values. Although the travel times depend on the distance, on the very same day, it may be stochastic due to incidents or various factors that obstruct travel. Owing to this uncertainty, HCPP has been solved within the scope of this paper using the chance-constrained stochastic programming method. The objective function coefficients which constitute the randomness in the chance-constrained model are supposed to be compliant with a normal distribution. The suggested model was only able to find a solution for small-sized samples due to the NPhard nature of the problem. Two new approaches, one of which is heuristic and the other meta-heuristic, are proposed through the use of Matlab-2019b programming language to solve sixty test instances 
addressed in the purview of the research to identify solutions on the proper time to medium and large problems. Both of the methods proposed in the research were not used for the HCPP solution in previous studies. The proposed heuristics is based on GSA. The current meta-heuristic is a different method from the ACO, although it is built on it. As can be seen in Table 3, Table 4 and Table 5 although the ACO-based meta-heuristic algorithm finds better results in all test instances, the average time used by the GSA-based heuristic less than the ACO-based meta-heuristic algorithm. These results are presented in Fig. 6 and Fig. 7. One possible explanation for this is that the meta-heuristic, unlike the heuristic, searches all space for the solution.

\section{Conclusion}

The Stochastic Travel Time (STT-HCPP) version of HCPP, a CPP variant, is examined in this research. In this problem, where travel times between nodes are uncertain, the objective is to find the shortest tour or tours by visiting all the edges of the network at least once. The stochastic analysis of travel times is significant in terms of meeting real-life situations. A similar study on HCPP has not been encountered in the literature. In this framework, the mathematical model of HCPP was created through a chanceconstrained stochastic programming approach, but due to the NP nature of the problem, optimal solutions to large-size problems could not be achieved. It has therefore become necessary to utilize heuristic algorithms to solve the problem. GSA and ACO based techniques have been implemented to solve the problem. The suggested algorithms were tested by using the test problems stated by Çodur and Yilmaz (2020) and their effectiveness was compared. The algorithms suggested on the basis of these results offer suitable solutions in a short time, including for large-size problems.

For further research, several examples can be illustrated. It is appropriate to discuss cases in which stochastic travel times suit various distributions (such as triangular, gamma, etc.). The meta-heuristic algorithm established can be evaluated under various parameter values. Different heuristic and/or metaheuristic approaches can be developed for STT-HCPP. The solutions obtained can be compared with the ones obtained in this study.

Funding Statement: The authors received no specific funding for this study.

Conflicts of Interest: The authors declare that they have no conflicts of interest to report regarding the present study.

\section{References}

Aksaraylı, M., Pala, O. (2015). Şans kisıtll stokastik programlama yaklaşımı ile ofis ürünleri üretim sistemi modellemesi. 15. Üretim Araştırmaları Sempozyumu.

Alfa, A. S., Liu D.Q. (1988). Postman routing problem in a hierarchical network. Engineering Optimization, 14, 127 138.

Atalay, K.D., Apaydın, A. (2011). Şans kısıtlı stokastik programlama problemlerinin deterministik eşitlikleri. Anadolu Üniversitesi Bilim ve Teknoloji Dergisi, 1, 1-18.

Binart, S., Dejax, P., Gendreau, M., Semet, F. (2016). A 2-stage method for a field service routing problem with stochastic travel and service times. Computers \& Operations Research, 65, 64-75.

Cabral, E.A., Gendreau M., Ghiani G., Laporte G. (2004). Solving the hierarchical Chinese postman problem as a rural postman problem. European Journal of Operational Research, 155, 44-50.

Carraway, R. L., Morin, T. L., Moskowitz, H. (1989). Generalized dynamic programming for stochastic combinatorial optimization. Operations Research, 37, 819-829.

Chang. T., Wan Y., Tsang W. (2009). A stochastic dynamic traveling salesman problem with hard time Windows. 
European Journal of Operational Research, 198,748-759.

Charnes, A., Cooper, W. W. (1959). Chance-constrained programming. Management Science, 6, 73-79.

Çodur, M. K., Yılmaz, M. A (2020). Time-dependent hierarchical Chinese postman problem. Central European Journal of Operations Research, 28, 337-366.

Colombi, M., Corberan A., Mansini R., Plana I., Sanchis J.M. (2017). The hierarchical mixed rural postman problem: polyhedral analysis and a branch-and-cut algorithm. European Journal of Operational Research, 257, 1-12.

Corberan, A., Marti R., Sanchis J. M.. A. (2002). Grasp heuristic for the mixed Chinese postman problem. European Journal of Operational Research, 142, 70-80.

Damodaran, P., Krishnamurthi M., Srihari K. (2008). Lower bounds for hierarchical Chinese postman problem. International Journal of Industrial Engineering: Theory, Applications and Practice, 15, 36-44.

Dorigo, M., Socha, K. (2007). An introduction to ant colony optimization. IRIDIA, Bruxelles, technical report series. Dror, M., Stern H., Trudeau P. (1987). Postman tour on a graph with precedence relation on arcs. Networks, 17, 283294.

Dutta, J., Barma, P.S., Kar, S., De, T. (2019). A modified kruskal's algorithm to improve genetic search for open vehicle routing problem. International Journal of Business Analytics (IJBAN), 6, 55-76.

Ehmke, J. F., Campbell, A. M., Urban, T. L. (2015). Ensuring service levels in routing problems with time windows and stochastic travel times. European Journal of Operational Research, 240, 539-550.

Errico, F., Desaulniers, G., Gendreau, M., Rei, W., Rousseau, L. M. (2016). The vehicle routing problem with hard time windows and stochastic service times. EURO Journal on Transportation and Logistics, 7.

Errico, F., Desaulniers, G., Gendreau, M., Rei, W., Rousseau, L. M. (2016). A priori optimization with recourse for the vehicle routing problem with hard time windows and stochastic service times. European Journal of Operational Research, 249, 55-66.

Gendreau, M., Laporte, G., Séguin, R. (1996). Stochastic vehicle routing. European Journal of Operational Research, 88, 3-12.

Ghiani, G., Improta G. (2000). An algorithm for the hierarchical Chinese postman problem. Operations Research Letters, 26, 27-32.

Gutierrez, A., Dieulle, L., Labadie, N., Velasco, N. (2016). A multi population memetic algorithm for the vehicle routing problem with time windows and stochastic travel and service times. IFAC-PapersOnLine, 49(12), 1204-1209.

Higle, J.L. (2005). Stochastic programming: Optimization when uncertainty matters. In Emerging Theory, Methods, and Applications; Informs: Catonsville, MD, USA, pp. 30-53.

Hou, L., Zhou, H. (28-31 May 2010). Stochastic vehicle routing problem with uncertain demand and travel time and simultaneous pickups and deliveries. 2010 Third International Joint Conference on Computational Science and Optimization, Huangshan, Auhui, China.

Hulsurkar, S., Biswal M.P., Sinha, S.B. (1997). Fuzzy programming approach to multi-objective stochastic linear programming problems. Fuzzy Sets and Systems, 88,173-181.

Jula, H., Dessouky, M., Ioannou, P. (2006). Truck Route Planning in Non-Stationary Stochastic Networks with TineWindows at Customer Locations. IEEE Transactions on Intelligent Transportation Systems, 37, 51-63.

Kao, E. P. A. (1978). Preference order dynamic program for a stochastic traveling salesman problem. Operations Research, 26, 1033-1045.

Kapukaya, O. (2019). Design of self tuning pid controller using adaptive particular swarm optimization and ant colony optimization (M.Sc. Thesis), Kahramanmaraş Sütçü Imam University, Kahramanmaraş, 2019.

Kenyon, A. S., Morton, D. P. (2003). Stochastic vehicle routing with random travel times. Transportation Science, 37, 69-82.

Kim, S., Lewis, M. E., White, C. C. (2005). Optimal vehicle routing with real-time traffic information, IEEE Transactions on Intelligent Transportation Systems, 4, 178-188.

Kızıloğlu, K. (2017). The heuristic solution approach for stochastic demand multidepot vehicle routing problem (M. Sc. Thesis), Gazi University, Ankara.

Korteweg, P., Volgenant T. (2006). On the hierarchical Chinese postman problem with linear ordered classes. European Journal of Operational Research, 169, 41-52. 
Laporte, G., Gendreau, M., Potvin, J. Y., Semet, F. (2000). Classical and modern heuristics for the vehicle routing problem. International transactions in operational research, 7, 285-300.

Laporte, G., Louveaux, F., Mercure, H. (1992). The vehicle routing problem with stochastic travel times. Transportation Science, 26, 161-170.

Li, X., Tian, P., Leung, S. C. (2010). Vehicle routing problems with time windows and stochastic travel and service times: Models and algorithm. International Journal of Production Economics, 125, 137-145.

Majumder, S., Kar, S., Pal, T. (2019). Uncertain multi-objective Chinese postman problem. Soft Computing, 23, 11557-11572.

Majumder, S., Kundu, P., Kar, S., Pal, T. (2019). Uncertain multi-objective multi-item fixed charge solid transportation problem with budget constraint. Soft Computing, 23, 3279-3301.

Miranda, D. M., Branke, J., Conceição, S. V. Algorithms for the multi-objective vehicle routing problem with hard time windows and stochastic travel time and service time. Applied Soft Computing. 2018, 70, 66-79.

Miranda, D. M.; Conceição, S. V. (2016). The vehicle routing problem with hard time windows and stochastic travel and service time. Expert Systems with Applications, 64, 104-116.

Mukherjee, A., Panigrahi, G., Kar, S., Maiti, M. (2019). Constrained covering solid travelling salesman problems in uncertain environment. Journal of Ambient Intelligence and Humanized Computing, 10, 125-141.

Nguyen, V. A., Jiang, J., Ng, K. M., Teo, K. M. (2016). Satisficing measure approach for vehicle routing problem with time windows under uncertainty. European Journal of Operational Research, 248, 404-414.

Oyola, J., Arntzen, H., Woodruff, D. L. (2018). The stochastic vehicle routing problem, a literature review, part I: models. EURO Journal on Transportation and Logistics, 7, 193-221.

Russell, R. A., Urban, T. L. (2008). Vehicle routing with soft time windows and Erlang travel times. Journal of the Operational Research Society, 59, 1220-1228.

Sayata, U.B., Desai N.P. (12-13 June 2015). An algorithm for Hierarchical Chinese postman problem using minimum spanning tree approach based on Kruskal's algorithm. IEEE International Advance Computing Conference (IACC), Banglore, India.

Shen, Z., Ordóñez, F., Dessouky, M. The stochastic vehicle routing problem for minimum unmet demand. In Optimization and logistics challenges in the enterprise, W. Chaovalitwongse., K. C. Furman., P. M. Pardalos., Eds.; In Springer Optimization and Its Applications, Springer: Boston, US. 2009; volume 30, pp. 349-371.

Tadei, R., Perboli, G., Perfetti, F. (2017). The multi-path traveling salesman problem with stochastic travel costs. EURO Journal on Transportation and Logistics, 6, 3-23.

Taha, H.A. (2007). Operations Research an Introduction. 7th ed.; Prentice Hall, NJ, United States.

Tan, G., Cui, X., Zhang, Y. (23-28 October 2005). Chinese postman problem in stochastic networks. In Joint International Conference on Autonomic and Autonomous Systems and International Conference on Networking and Services-(icas-isns' 05), Papeete, Tahiti, French Polynesia.

Taş, D., Dellaert, N., Van Woensel, T., De Kok, T. (2013). Vehicle routing problem with stochastic travel times including soft time windows and service costs. Computers \& Operations Research, 40, 214-224.

Uğurlu, O. (2018). Algorithmic approaches for routing problems, (Ph. D. Thesis), Ege University, İzmir.

$\mathrm{Xu}$, A., Viriyasuthee, C., Rekleitis, I. (2014). Efficient complete coverage of a known arbitrary environment with applications to aerial operations. Autonomous Robots, 36, 365-381.

Y1lmaz, M. (2018). Route management for vehicles used in road maintenance activities through hierarchical chinese postman problem approach. Journal of the Institute of Science and Technology, 8, 107-115.

Zhang, B., Peng, J. (2012). Uncertain programming model for Chinese postman problem with uncertain weights. Industrial Engineering \& Management Systems, 11, 18-25.

Zhang, J., Lam, W. H., Chen, B. Y. (2013). A stochastic vehicle routing problem with travel time uncertainty: tradeoff between cost and customer service. Networks and Spatial Economics, 13, 471-496. 